\title{
Lattice Boltzmann simulations of particle-laden liquid bridges: Effects of volume fraction and wettability
}

\author{
Kevin W. Connington ${ }^{1}$, Marc Z. Miskin ${ }^{4}$, Taehun Lee ${ }^{2}$, Heinrich M. \\ Jaeger $^{4}$, and Jeffrey F. Morris*1,3 \\ ${ }^{1}$ The Levich Institute, The City College of New York, New York, NY, 10031 \\ ${ }^{2}$ The Department of Mechanical Engineering, The City College of New York, New York, NY, \\ 10031 \\ ${ }^{3}$ The Department of Chemical Engineering, The City College of New York, New York, NY, \\ 10031 \\ ${ }^{4}$ The James Franck Institute, The University of Chicago, Chicago, IL, 60637
}

April 25, 2015

\begin{abstract}
The influence of particles on the dynamics and eventual rupture of stretching liquid bridges is demonstrated experimentally in a drop-forming case. To analyze the particle-scale basis for the influence of particles in this flow, a lattice Boltzmann algorithm for a three-phase system of liquid, gas, and solid particles, has been developed. This provides full coupling between particles and fluids, fluid interfacial forces, and possible entry of particles into the interface (i.e. full and partial wetting by the liquid are considered). This work details the numerical method and its validation, and presents results of the simulations and related experiments. Fullywetting particles up to a solid volume fraction of $\phi=0.3$ monotonically increased the rupture length of liquid bridges, as seen in experiments; experimental results show that the increase continues to a maximum at $\phi \approx 0.4$, a condition beyond the present numerical capability. Depending on the wettability and volume fraction of the particles in the liquid bridge, particles can alter the structure of the bridge at pinch-off, suppress satellite drops, or produce asymmetrical pendant/sessile suspension drops as a result of their discrete nature. Particles with a neutrally wetting contact angle $\left(\theta=90^{\circ}\right)$ can reside in the bulk or be immersed in the interface if fluid deformation brings them into contact with it, and capillary forces are found to bring
\end{abstract}

${ }^{*}$ Corresponding author; email: morris@ccny . cuny.edu 
the interfacial particles near the narrow region (or "throat") of the bridge prior to rupture. Fully wetting particles $\left(\theta \approx 0^{\circ}\right)$ remained interior to the liquid bridge, leaving less space to escape the throat region. Neutrally wetting particles increased the rupture length and altered the pinch-off structure relative to the particle-free case, but less so than fully wetting particles.

Keywords lattice Boltzmann; liquid bridge; particle suspension; interface

\section{Introduction}

Solid particles suspended in a fluid alter the behavior of many physical flow situations relative to the particle-free case. The particles resist deformation in bulk fluid regions, leading to effective suspension properties distinct from the properties of the interstitial fluid [1]. Suspension flows that involve a free surface can also exhibit capillary forces due to particles which cause deformation of, or become embedded in, the interface. The wetting properties of the particles and the local deformation of the interface can result in surface tension forces that transport particles along the interface [2]. The stretching and rupture of a liquid column containing particles exhibits these complications. This is a complex situation, but one that has increasingly important applications in jet stability and drop formation which arise in the printing of particle-laden ceramics, textiles, electronics, and inks [3-6] as well as biological cells [7]. In this work, we focus on the well-controlled case of stretching a particle-laden liquid bridge.

There has been extensive effort devoted to understanding the physics related to the rupture of a particle-free liquid bridge, as summarized by Eggers [8]. A liquid bridge is stretched in one of two typical situations. When a drop is extruded from a nozzle, gravity forces overcome the surface tension forces adhering the bulk of the drop to the nozzle. As the drop falls away, a thin filament connects the bulk of the drop to the liquid still being extruded from the nozzle. The other mode of stretching occurs when a volume of fluid simultaneously wets two solid objects that are pulled apart. In either case, the bridge of liquid thins, or necks, and finally ruptures.

The rupture process of a Newtonian liquid is governed by a competition between surface tension, viscosity, and inertia. In general, the filament contraction is driven by surface tension, and is retarded by viscosity. Surface tension causes perturbations of a liquid cylinder to be unstable, thus initiating an exponential thinning process. Sufficiently close to rupture, the necking behavior of liquid bridges exhibits a universal self-similar character regardless of the mode of stretching. The minimum neck radius, $R_{m}$ of the liquid bridge scales as $R_{m} \sim \tau^{\alpha}$, where $\tau=t_{\text {breakup }}-t>0$ with $t_{\text {breakup }}$ the time when breakup, or rupture, occurs, $t$ the time. The exponent $\alpha$ corresponds to different regimes. For inertia-dominated flows (the inviscid regime), $\alpha=2 / 3$ [8], and for viscosity-dominated flows, $\alpha=1$ [9]. There is a critically small thickness of the thread where both viscosity and inertia take on similar importance [8]. Viscosity becomes more important for inertia- 
dominated flows as the dimensions of the thread become small. Capillary pressure forces related to inertia also take on added significance for viscous-dominated flows because of the large curvature associated with the thin thread. Any bridge will sample this regime just prior to rupture, where $\alpha=1$, which is the same as the viscous dominated-regime, but with a different constant of proportionality.

There has been little work, in comparison, analyzing stretching liquid bridges with particles. Furbank \& Morris [10,11] performed experiments in which neutrally-buoyant (density-matched) particle-laden liquids were extruded from a nozzle, at a range of particle volume fractions, $0<\phi \leq 0.4$. They demonstrated that the addition of noncolloidal particles at large concentrations in this drop-forming process slowed the neck thinning far from rupture, as expected from increase of effective viscosity. While this generally increased the time to rupture, the granularity of the material was associated with a second stage of thinning which was localized along the axis of the filament and was notably more rapid than in a similar-viscosity pure liquid. The pinch-off structure was found to be qualitatively different when comparing a suspension to a pure liquid with viscosity equal to that of the suspension. Based on these observations, Furbank and Morris proposed a two-stage pinch-off process where particle effects are governed by bulk properties (effective viscosity) followed by one where the discrete size effects of the particles dominate. This and subsequent experimental work of which we are aware has been carried out with fully-wetting particles, meaning the contact angle of the suspending liquid with the particle surface is close to zero.

Bonnoit et al. [12] extended the investigation to address final detachment and observed thinning in three regimes. The previously mentioned effective viscosity regime transitions to a regime that behaves like the interstitial fluid. This happens when the volume fraction in the neck is very low, meaning the particles do not interact with the thinning fluid. Finally, there is a transition to an accelerated regime just before rupture. Van Deen et al. [13] studied systems with very low volume fraction and concluded that the accelerated regime begins when a particle in the thread directly interacts with the thinning dynamics. In this regime, thinning proceeds faster than a pure fluid with either effective or interstitial viscosity. Miskin \& Jaeger [14] demonstrated the minimum radius scaling $R_{m} \sim \tau^{2 / 3}$ for drops with large volume fractions of particles. They argued that the scaling results from particles forming bulges in the neck, creating a Laplace pressure that must be balanced by inertia. Bertrand et al. [15] studied the effects of volume fraction on the drop shape at pinch-off. Roché et al. [16] showed that concentration fluctuations in the thread can arise due to heterogeneous particle rearrangements directly linked to the onset of rupture. Furbank \& Morris [10] demonstrated that satellite drops at rupture are suppressed by adding particles.

The noted experimental work makes clear a range of behaviors occurs, depending on the properties of the interstitial fluid, combined with the size relative to the size of the nozzle and solid fraction $\phi$. To gain further insight to this complex process, analytical and numerical methods have been applied. Hameed \& Morris [17] developed a matched 
asymptotic expansion model that was solved by an implicit finite difference scheme. They used their model to investigate the influence of a single particle in the thread using a symmetric force dipole (known as a stresslet) to represent the particle. McIlroy \& Harlen [18] developed a one-dimensional model for an axisymmetric particle-laden bridge stretched between parallel plates, where point particles (smaller than the grid) were advected by the fluid, and their local concentration determined the local viscosity as a feedback mechanism. Although this model predicts quite acceptably the thinning behavior and variations in local particle density, it does not represent the finite size of the particles, and cannot predict aspects of pinch-off when the thread approaches the size of the particles.

To address the issues of finite-size particles in drop-forming and related processes, we have developed a numerical approach that captures both the fluid-fluid interface and particle-fluid interactions. This approach is based on the lattice Boltzmann method (LBM), which has become a widely-used tool for solving continuum scale fluid flows. Of most relevance here, there has been success using the LBM to simulate flows with multiple fluid components [21] and suspended particles [20,41], two aspects of the problem of particle-laden interfacial flows. In this method, the full Navier-Stokes equations, inclusive of both inertial and viscous effects, are recovered in the fluid domain, where flows in the liquid and ambient gas are solved in three dimensions. Finite-sized particles are transported by Newtonian dynamics, where a full two-way coupling is accounted for, meaning particles are transported due to pressure, viscous, and surface tension forces applied by the fluid at their boundaries. The resulting traction forces on the fluid in turn affect the fluid flow. This provides a unique and powerful tool to gain insight into the complicated behaviors of particle-laden interfacial flows. In this work, we study specifically the effects of particle wettability and solid fraction for a suspension bridge stretched between parallel plates. We analyze the rupture length and pinch-off structure for suspensions that use fully wetting (contact angle $\left.\theta \approx 0^{\circ}\right)$ and neutrally wetting $(\theta=$ $\left.90^{\circ}\right)$ particles. We further discuss the suppression of satellite drops and the post-rupture asymmetry of the volumes resulting from bridge rupture.

The rest of the paper is organized as follows. The numerical method is described in Sec.2; fuller details are available elsewhere [19]. The code is validated for the particle-free stretching liquid bridge with comparison to experiment in Sec.3. Results of our lattice Boltzmann simulations and the experimental studies of this work are discussed in Sec.4. Concluding remarks are given in Sec.5.

\section{Numerical Method}

The numerical method consists of a multi-component lattice Boltzmann algorithm for the fluid, coupled with Newtonian dynamics for finite-sized particles. The fluid evolves according to the discrete Boltzmann equation, inclusive of surface tension forces at fluid interfaces. Imposed boundary conditions for the distribution function recover the no 
slip condition on solid surfaces, but allow for diffusion of the contact line to avoid a stress singularity. Given the location and velocities of solid boundaries at a particular time, the first aspect of the coupling is set. The evolution of the distribution functions will recover the pressure evolution equation (effectively the continuity equation), the momentum equation, and the Cahn-Hilliard equation for the composition. The boundary conditions in turn impart pressure, viscous, and surface tension forces on the particles, effecting the second aspect of the coupling, where particles are transported according to Newtonian dynamics. Here, we describe the two separate aspects of the algorithm, the multi-component algorithm and the particle dynamics algorithm, and highlight important points. We refer the reader to other work Connington et al. [33] for further development.

\subsection{Multi-component Algorithm}

We implement the multi-component algorithm developed by Lee \& Liu [21], which is a free energy-based diffuse interface method. This method has been shown to suppress spurious currents, allowing simulation of two immiscible fluids with a large density ratio.

In the Cahn-Hilliard equation [22], a system of binary fluids evolves so that the total free energy is minimized. The system evolves due to gradients of a computed chemical

potential, $\mu$, i.e., the specific free energy. The total free energy contains both local and nonlocal terms, as well as contributions from solid boundaries:

$$
\begin{aligned}
\Psi=\Psi_{b}+\Psi_{s} & =\int_{V}\left(E_{0}(C)+\frac{\kappa}{2}|\nabla C|^{2}\right) \mathrm{d} V \\
& +\int_{S}\left(\phi_{0}-\phi_{1} C_{S}+\phi_{2} C_{S}^{2}-\phi_{3} C_{S}^{3}+\cdots\right) \mathrm{d} S .
\end{aligned}
$$

Here $\Psi$ is the total free energy, and $\Psi_{b}$ and $\Psi_{s}$ represent contributions from the bulk fluids and solid boundaries, respectively. The local contribution to the bulk free energy is $E_{0}=\beta C^{2}(C-1)^{2}$, where $\beta$ is a constant, and $C \in[0: 1]$ is the composition, indicating the local concentration of each species. Nonlocal effects are characterized by $\kappa$. The composition at the surface is $C_{s}$, and the $\phi_{i}$ are constant coefficients. Given the composition, also known as the order parameter, the mass density can be obtained by $\rho=C \rho_{1}+(1-C) \rho_{2}$, where $\rho_{1}$ and $\rho_{2}$ are the bulk densities of the individual components.

The path the system takes toward its minimum energy configuration is governed by the Cahn-Hilliard equation:

$$
\frac{\partial C}{\partial t}+\nabla \cdot(\boldsymbol{u} C)=M \nabla^{2} \mu
$$

where $\boldsymbol{u}$ is the volume averaged velocity, and $M$ is the constant mobility, a numerical parameter. The chemical potential is obtained from the total free energy, upon variation [22], and is written $\mu=\mu_{0}-\kappa \nabla^{2} C$, where $\mu_{0}=\partial E_{0} / \partial C$ is the classical (local) part of 
the chemical potential. Terms from the surface integral are included in the definition of $\mu$ when computing $\nabla^{2} C$ near a boundary [33]. The system ceases to evolve when $\mu$ is constant; this can be a true or quasi-equilibrium state, depending on whether the free energy minimum is global or local, respectively.

Numerically, the local term of eq.(1) is minimized when the components are completely separated. On the other hand, the nonlocal term is minimized when the species are completely mixed. The competition between these two terms results in a nearly complete separation of components, except for a finite-sized interfacial region. The constants $\beta$ and $\kappa$ indicate the relative importance of the local and nonlocal parts of the bulk free energy, determining the size of the interface thickness. The choice $\kappa=\beta \xi^{2} / 8$ results in an interfacial thickness $\xi$ with a profile $C(z)=0.5+0.5 \tanh (2 z / \xi)$, where $z$ is the coordinate normal to the interface. As another consequence of choosing the free energy parameters, the liquid-gas surface tension is specified by $\sigma=\sqrt{2 \kappa \beta} / 6$. The values of $\beta$ and $\kappa$ can be chosen to specify the surface tension and interface thickness desired for a simulation.

The solution to (2) requires two boundary conditions. The boundary condition for $\nabla^{2} \mu$ is $\left.\boldsymbol{n} \cdot \nabla \mu\right|_{s}=0$, where $\boldsymbol{n}$ is the unit vector normal to the surface; this prevents a mass flux normal to a solid boundary. The boundary condition for $\nabla^{2} C$ is established by minimizing the surface free energy. We use the cubic boundary condition which specifies the parameter set: $\phi_{0}=\phi_{1}=0, \phi_{2}=\phi_{c} / 2$, and $\phi_{3}=\phi_{c} / 3$, where $\phi_{c}$ is chosen to recover the desired contact angle at equilibrium [23,24]. Given these choices, the boundary condition for $\nabla^{2} C$ becomes $\left.\boldsymbol{n} \cdot \nabla C\right|_{s}=\frac{\phi_{c}}{\kappa}\left(C_{s}-C_{s}^{2}\right)$.

To recover the equations for conservation of mass and momentum, we begin with the discrete Boltzmann equation (DBE) for the transport of binary fluids [25-29]:

$$
\frac{\partial f_{\alpha}}{\partial t}+\boldsymbol{e}_{\boldsymbol{\alpha}} \cdot \boldsymbol{\nabla} \boldsymbol{f}_{\boldsymbol{\alpha}}=-\frac{1}{\lambda}\left(f_{\alpha}-f_{\alpha}^{e q}\right)+\frac{1}{c_{s}^{2}}\left(\boldsymbol{e}_{\alpha}-\boldsymbol{u}\right) \cdot \boldsymbol{F} \Gamma_{\alpha} .
$$

Here $f_{\alpha}$ is the distribution function corresponding to the discretized velocity $\boldsymbol{e}_{\alpha}, c_{s}$ is the speed of sound, $\lambda$ is the relaxation time, $\boldsymbol{F}$ is the intermolecular force recovering surface tension effects, $\Gamma_{\alpha}(\boldsymbol{u})=f_{\alpha}^{e q} / \rho$, and $f_{\alpha}^{e q}$ is the equilibrium distribution function defined by $f_{\alpha}^{e q}=t_{\alpha} \rho\left[1+\left(\boldsymbol{e}_{\alpha} \cdot \boldsymbol{u}\right) / c_{s}^{2}+\left(\boldsymbol{e}_{\alpha} \cdot \boldsymbol{u}\right)^{2} /\left(2 c_{s}^{4}\right)-(\boldsymbol{u} \cdot \boldsymbol{u}) /\left(2 c_{s}^{2}\right)\right] ; t_{\alpha}$ is the weight corresponding to $\boldsymbol{e}_{\alpha}$.

For stability reasons, the DBE for mass and momentum is transformed into the DBE for pressure evolution and momentum $[30,31]$ by defining a new distribution function $g_{\alpha}=f_{\alpha} c_{s}^{2}+\left(p-\rho c_{s}^{2}\right) \Gamma_{\alpha}(0)$ :

$$
\frac{\partial g_{\alpha}}{\partial t}+\boldsymbol{e}_{\alpha} \cdot \nabla g_{\alpha}=-\frac{1}{\lambda}\left(g_{\alpha}-g_{\alpha}^{e q}\right)+\left(\boldsymbol{e}_{\alpha}-\boldsymbol{u}\right) \cdot\left[\nabla \rho c_{s}^{2}\left(\Gamma_{\alpha}-\Gamma_{\alpha}(0)\right)+\mu \nabla C \Gamma_{\alpha}\right] .
$$

From (4) we recover the pressure evolution and momentum equations: 


$$
\begin{aligned}
\frac{\partial p}{\partial t}+\rho c_{s}^{2} \nabla \cdot \boldsymbol{u} & =0, \text { and } \\
\rho\left(\frac{\partial \boldsymbol{u}}{\partial t} \boldsymbol{u} \cdot \nabla \boldsymbol{u}\right) & =-\nabla p+\mu \nabla C+\nabla\left[\rho c_{s}^{2} \lambda \cdot\left(\nabla \boldsymbol{u}+(\nabla \boldsymbol{u})^{T}\right)\right] .
\end{aligned}
$$

The dynamic viscosity is identified as $\eta=\rho c_{s}^{2} \lambda$.

Another distribution function is introduced to track the order parameter: $h_{\alpha}=$ $(C / \rho) f_{\alpha}$ and $h_{\alpha}^{e q}=(C / \rho) f_{\alpha}^{e q}$. The corresponding DBE is written

$$
\frac{\partial h_{\alpha}}{\partial t}+\boldsymbol{e}_{\alpha} \cdot \nabla h_{\alpha}=-\frac{1}{\lambda}\left(h_{\alpha}-h_{\alpha}^{e q}\right)+M \nabla^{2} \mu \Gamma_{\alpha}+\left(\boldsymbol{e}_{\alpha}-\boldsymbol{u}\right) \cdot\left[\nabla C-\frac{C}{\rho c_{s}^{2}} \nabla p-\mu \nabla C\right] \Gamma_{\alpha}
$$

From (7) we recover

$$
\frac{\partial C}{\partial t}+\nabla \cdot(\boldsymbol{u} C)=M \nabla^{2} \mu
$$

which is the advective Cahn-Hilliard equation when $\nabla \cdot \boldsymbol{u}=0$. See [21] for the derivation of these equations and their Chapman-Enskog expansion.

The LBE's are obtained by integrating the DBE's, (4) and (7), along characteristics using a trapezoidal approximation over a time step $\delta t$. Modified distribution functions are introduced as in Lee \& Liu [21], and the resulting LBE's become:

$$
\begin{aligned}
\bar{g}_{\alpha}(\boldsymbol{x}, t)= & \bar{g}_{\alpha}\left(\boldsymbol{x}-\boldsymbol{e}_{\alpha} \delta t, t-\delta t\right)-\left.\frac{1}{\tau+1 / 2}\left(\bar{g}_{\alpha}-\bar{g}_{\alpha}^{e q}\right)\right|_{\left(\boldsymbol{x}-\boldsymbol{e}_{\alpha} \delta t, t-\delta t\right)} \\
& +\delta t\left(\boldsymbol{e}_{\alpha}-\boldsymbol{u}\right) \cdot\left[\nabla^{\mathrm{MD}} \rho c_{s}^{2}\left(\Gamma_{\alpha}-\Gamma_{\alpha}(0)\right)+\left(\mu \nabla^{\mathrm{MD}} C-\rho g_{y} \nabla H\right) \Gamma_{\alpha}\right]_{\left(\boldsymbol{x}-\boldsymbol{e}_{\alpha} \delta t, t-\delta t\right)}, \text { and }
\end{aligned}
$$

$$
\begin{aligned}
\bar{h}_{\alpha}(\boldsymbol{x}, t)= & \bar{h}_{\alpha}\left(\boldsymbol{x}-\boldsymbol{e}_{\alpha} \delta t, t-\delta t\right)-\left.\frac{1}{\tau+1 / 2}\left(\bar{h}_{\alpha}-\bar{h}_{\alpha}^{e q}\right)\right|_{\left(\boldsymbol{x}-\boldsymbol{e}_{\alpha} \delta t, t-\delta t\right)} \\
& +\left.\frac{\delta t}{2} M \nabla^{2} \hat{\mu} \Gamma_{\alpha}\right|_{\left(\boldsymbol{x}-\boldsymbol{e}_{\alpha} \delta t, t-\delta t\right)}+\left.\frac{\delta t}{2} M \nabla^{2} \hat{\mu} \Gamma_{\alpha}\right|_{(\boldsymbol{x}, t-\delta t)} \\
& +\delta t\left(\boldsymbol{e}_{\alpha}-\boldsymbol{u}\right) \cdot\left[\nabla^{\mathrm{MD}} C-\frac{C}{\rho c_{s}^{2}}\left(\nabla^{\mathrm{MD}} p-\mu \nabla^{M D} C-\rho g_{y} \nabla H\right) \Gamma_{\alpha}\right]_{\left(\boldsymbol{x}-\boldsymbol{e}_{\alpha} \delta t, t-\delta t\right)}
\end{aligned}
$$

The relaxation time $\tau=\lambda / \delta t$ is related to the kinematic viscosity by $\nu=\tau c_{s}^{2} \delta t$, and is prescribed by $1 / \tau=C / \tau_{1}+(1-C) / \tau_{2}$, where $\tau_{1}$ and $\tau_{2}$ correspond to the bulk kinematic viscosities of the respective fluids. Gravity is denoted by $g_{y}$, and the term $\nabla H$ is the gradient of the height, which is just a unit vector in the vertical direction. 
The macroscopic variables are computed from respective moments of the distribution function:

$$
\begin{aligned}
C & =\sum_{\alpha} \bar{h}_{\alpha}, \\
\rho \boldsymbol{u} & =\sum_{\alpha} \boldsymbol{e}_{\alpha} \bar{g}_{\alpha}+\frac{\delta t}{2} \mu \nabla^{C D} C, \\
p & =\bar{g}_{\alpha}+\frac{\delta t}{2} \boldsymbol{u} \cdot \nabla^{\mathrm{CD}} \rho c_{s}^{2} .
\end{aligned}
$$

The superscript labels $M D$ and $C D$ refer to mixed difference and central difference gradients; see Lee \& Liu for details [21].

\subsection{Particle Dynamics}

The presence of the particles requires the specification of boundary conditions for the fluid near a solid boundary as well as the prescription of forces acting between the fluid and the particles. Consider Fig.1 where a curved boundary (particle) cuts the lattice. We employ a standard bounce-back-on-the-link (BBL) scheme where a distribution function, $\bar{g}_{\alpha}$ or $\bar{h}_{\alpha}$, at $\boldsymbol{x}_{f}$ streams toward the particle, hits the wall located half-way along the link $\left(\boldsymbol{x}_{b}\right)$ connected to the interior solid node $\boldsymbol{x}_{s}$, and arrives back at the node it originated from, now as $\bar{g}_{\bar{\alpha}}$ or $\bar{h}_{\bar{\alpha}}$. The scheme must be augmented to account for moving walls [20], and is written:

$$
\begin{aligned}
& \bar{g}_{\bar{\alpha}}=\bar{g}_{\alpha}+2 \rho t_{\alpha}\left(\boldsymbol{u}_{b} \cdot \boldsymbol{e}_{\bar{\alpha}}\right), \text { and } \\
& \bar{h}_{\bar{\alpha}}=\bar{h}_{\alpha}+2 \frac{C}{c_{s}^{2}} t_{\alpha}\left(\boldsymbol{u}_{b} \cdot \boldsymbol{e}_{\bar{\alpha}}\right) .
\end{aligned}
$$

The boundary node velocity, $\boldsymbol{u}_{b}$, can be computed from the particle position and velocity.

Forces from the fluid cause particle motion: here we have pressure, viscous, and surface tension forces. Pressure and viscous forces are calculated using the momentum exchange (ME) method [20]. For each application of the boundary condition from (14), a distribution function that hits a wall and bounces back effectively reverses its "momentum." In order to do this, an equal and opposite impulse of momentum must be applied to the particle. For each link $\alpha_{c}$ cut by the particle surface at $\boldsymbol{x}_{b}$, the scheme is written:

$$
\boldsymbol{F}\left(\alpha_{c} ; \boldsymbol{x}_{b}\right)=\left(\bar{g}_{\alpha}+\bar{g}_{\bar{\alpha}}\right) \boldsymbol{e}_{\alpha} .
$$

The total force on a particle is computed by taking the summation of all impulses: $\boldsymbol{F}_{T}=\sum_{x_{b}} \sum_{\alpha_{c}} \boldsymbol{F}\left(\alpha_{c} ; \boldsymbol{x}_{b}\right)$. The torque on the particle is obtained by taking moments of the force impulses about the particle center of mass. 


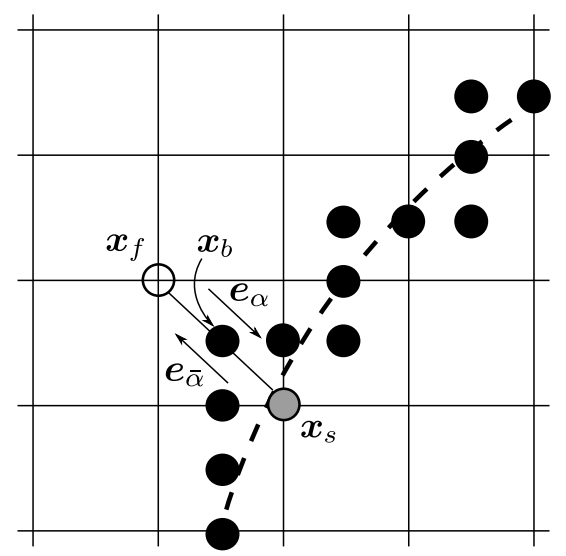

Figure 1: A representation of a curved boundary mapped onto the lattice. The dashed line represents the theoretical boundary of a curved wall such as a particle. The actual boundary points, designated $\boldsymbol{x}_{b}$ (filled black circles), are located half-way along links that connect a fluid node $\boldsymbol{x}_{f}$ (open circle) to an interior solid node $\boldsymbol{x}_{s}$ (filled grey circle) along a direction $\boldsymbol{e}_{\alpha} \delta t$. The direction $\boldsymbol{e}_{\bar{\alpha}} \delta t$ correspondingly connects the solid node to the fluid node. The $\delta t$ 's were omitted from the figure for clarity.

The particle dynamics, i.e. the update of the particle position and velocity, is unstable if $\bar{g}_{\bar{\alpha}}$ is computed explicitly using the particle velocity of the current time step. A stable scheme is achieved if the particle velocity at the next time step is calculated implicitly, and the resulting velocity used to compute the boundary velocity in (14) and (15). This requires two "sweeps" through the domain; the first sweep assembles the data needed to solve for the particle velocities implicitly, and the second sweep uses the new velocities to impose boundary conditions (14) and (15) [32].

Surface tension forces are of the form

$$
\boldsymbol{F}_{S T}=\oint_{\mathcal{C}} \sigma \boldsymbol{m} \mathrm{d} \ell
$$

where $\mathrm{d} \ell$ is a line element tangent to the three phase contact line $\mathcal{C}$ (a closed path), $\sigma$ is the surface tension, and $\boldsymbol{m}$ is a unit vector tangent to the interface locally (Fig. 2). A sweep through the domain (which can be performed at the same time as the ME sweep) is required to locate the contact line, discretize it, and compute the local tangent to the interface for each line element. The details of the algorithm are specified in Connington et al. [33]. The surface tension force is incorporated into the implicit algorithm as an external force. 


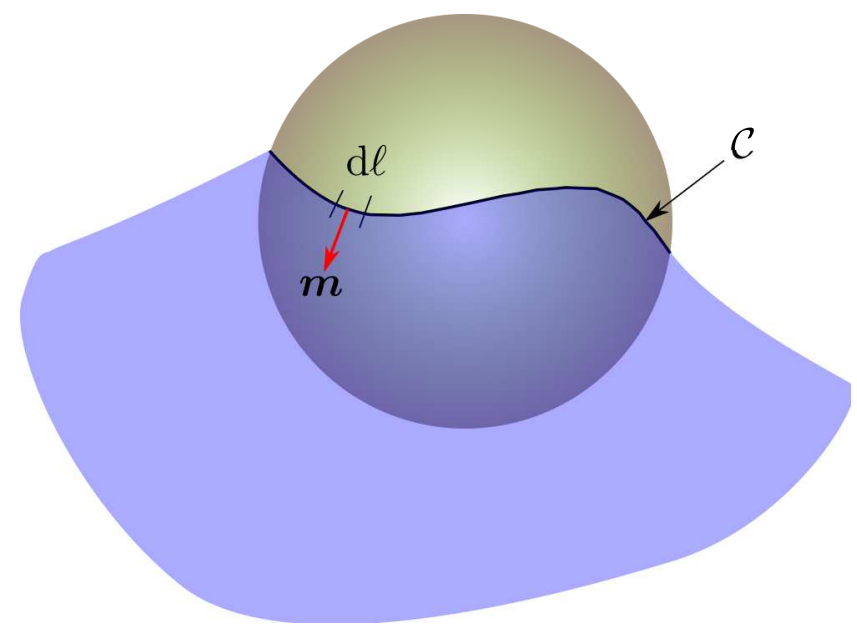

Figure 2: The three-phase contact line, $\mathcal{C}$, is depicted on the surface of a particle. Due to deformations associated with fluid motion, the form and location of the contact line are generally unknown.

\section{Code Validation}

Before presenting results for particles in stretching liquid bridges, we validate that the predicted liquid bridge behavior itself is correct. To do so, we compare our results to the experiment of Zhang et al. [36]. Consider the situation depicted in Fig. 3. A volume of fluid is held between two flat disks of radius $R$ separated by a distance $L$ (initial separation $L_{0}$ ). Gravity causes the shape of the bridge to be asymmetric, but both disks remain completely wetted. The upper disk is translated upward at a constant velocity $U$ while the lower disk is held fixed. The bridge necks as it elongates, eventually becomes unstable, and ruptures (Fig. 4). To validate our simulation, we compared our computed minimum bridge radius $\left(R_{m}\right)$ as a function of time to the experimental result.

The problem is specified by the dynamic viscosity and density of the liquid $\left(\eta_{l}, \rho_{l}\right)$ and gas $\left(\eta_{g}, \rho_{l}\right)$, respectively, the liquid-gas surface tension $(\sigma)$, disk radius $(R)$, initial disk separation $\left(L_{0}\right)$, volume of liquid $(V)$ contained between disks, velocity of upper disk $(U)$, and gravity $(g)$. To run a dynamically similar simulation, we must form seven dimensionless numbers from this parameter set, and match the simulation dimensionless numbers using parameters specified in lattice units to the experiment dimensionless numbers using parameters in physical units. The dimensionless groups we chose were the liquid-gas viscosity ratio $\left(\eta_{l} / \eta_{g}\right)$, liquid-gas density ratio $\left(\rho_{l} / \rho_{g}\right)$, initial separation aspect ratio $\left(L_{0} / R\right)$, the ratio of the liquid volume to the initial cylindrical volume between disks $\left(V /\left(\pi R^{2} L_{0}\right)\right)$, the Bond number $\left(B_{o}=\rho_{l} g R^{2} / \sigma\right)$, the Ohnesorge number $\left(O h=\eta_{l} / \sqrt{\rho_{l} \sigma R}\right)$, and the capillary number $\left(C a=\eta_{l} U / \sigma\right)$. For the simulation, we chose $\rho_{l}=1, U=0.0005$, and $R=100$ in lattice units, and used the dimensionless numbers to 


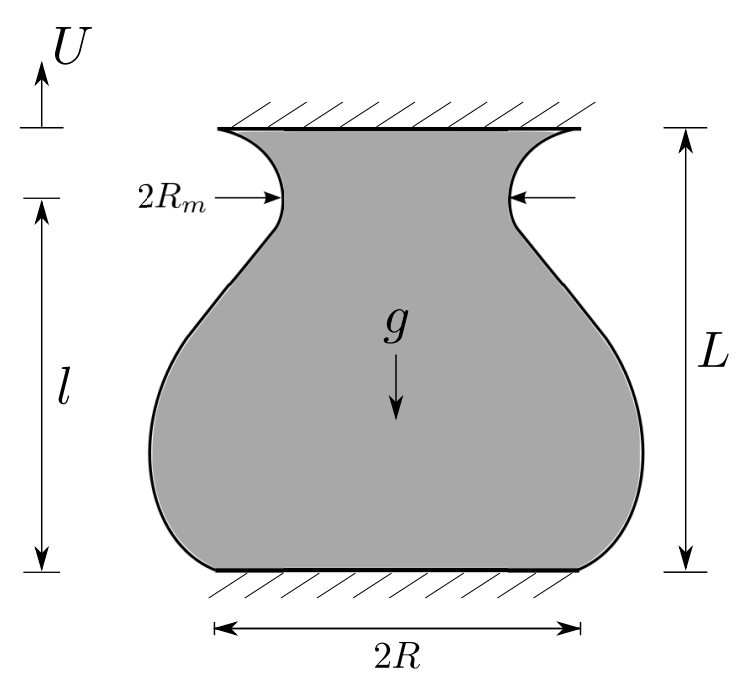

Figure 3: A liquid bridge without particles. The bottom disk of radius $R$ remained fixed while the upper disk translated upward with velocity $U$, against gravity $g$. The separation between disks is $L$. The minimum bridge radius is $R_{m}$, located at axial location $l$ measured from the bottom disk.

specify all remaining parameters. The full set of experimental and simulation parameters are listed in Table 1.

The initial profile of the bridge was not known a priori, so a generic shape containing the required volume of liquid was allowed to relax for 30,000 time steps before the disk motion was initiated. This was not enough time to completely eliminate fluid oscillation before the cylinder motion started, but the oscillation smoothed out sufficiently before the rupture stage.

Due to the discrete nature of the disk boundary conditions, a moving wall had to be approximated by a fixed layer of wall nodes with a prescribed normal velocity equal to the disk velocity. Only after $1 / U=2000$ lattice time steps would the wall make a discrete jump to the next lattice position, uncovering a layer of fluid nodes which had previously been inside the wall. When this occurred, new distribution functions were prescribed as equilibrium distribution functions. This procedure complicated a standard accounting of mass conservation. For the entire cycle of 2000 time steps between the wall jumping to the next position, the system lost a small amount of fluid mass each time step due to a flux of mass carried through the wall, prescribed by the velocity boundary condition. However, as the last step of the cycle created a new layer of fluid nodes, the total fluid mass in the system was nearly recovered by the end of a cycle. The overall scheme was very close to conserving mass when computed at the end of a wall layer generation cycle, where $99.36 \%$ of the original liquid mass remained after rupture. The scheme was not designed to conserve mass rigorously, so fluctuations were expected. For a case with such complicated boundary conditions, we consider this amount of mass loss to be acceptable, 

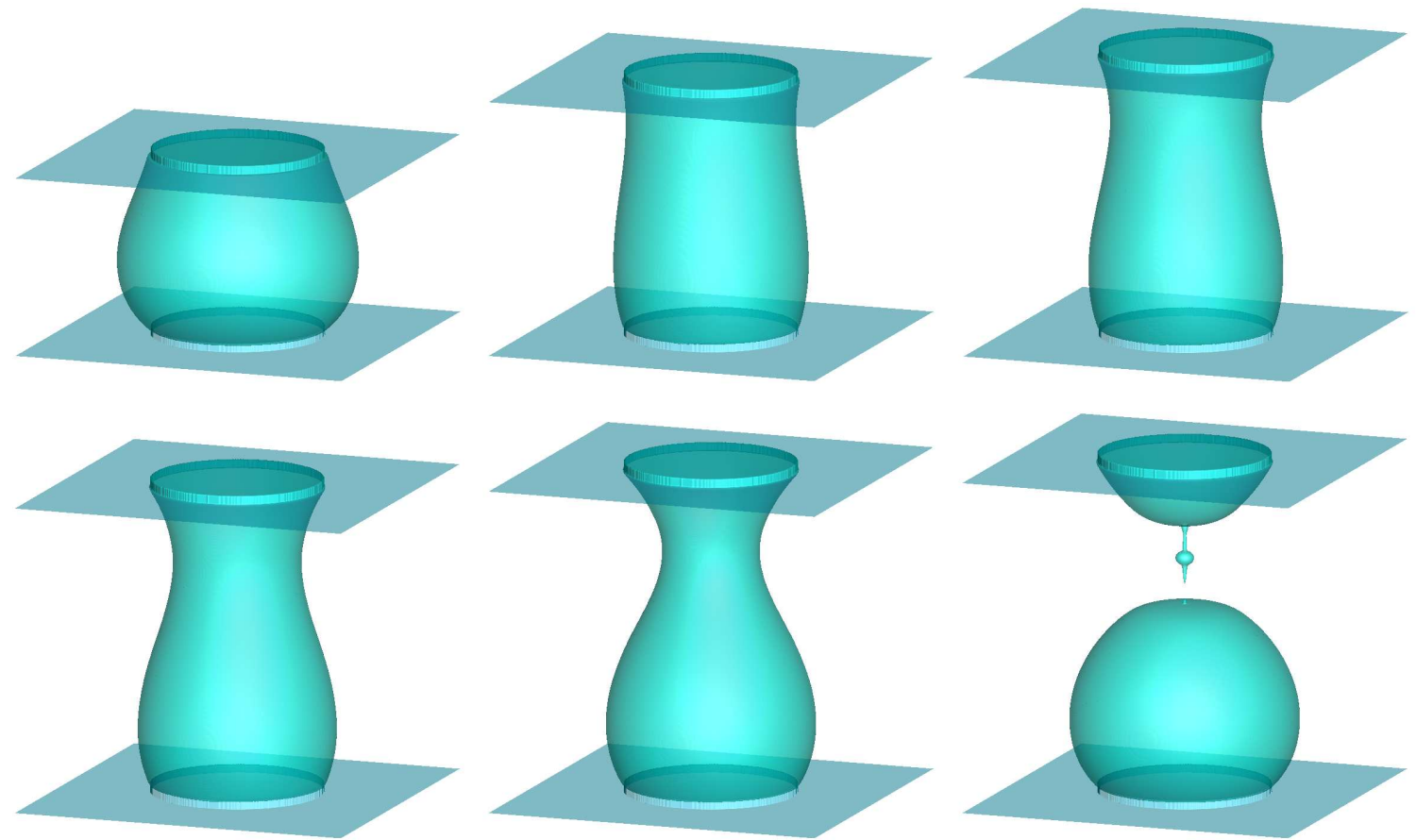

Figure 4: A sequence of images representing the dynamics of the stretching liquid bridge simulation. A volume of liquid was stretched between two circular disks. The contact radius of the liquid bridge is $0.16 \mathrm{~cm}$ and the initial column length is $0.32 \mathrm{~cm}$; the snapshots were taken at $t=0,240,320,400,453$, and $480 \mathrm{~ms}$, respectively, corresponding to lengths of $L=0.320,0.464,0.512,0.560,0.592$, and $0.608 \mathrm{~cm}$. The bridge necked as it was stretched, and eventually ruptured, producing a satellite drop. 


\begin{tabular}{|c|c|c|c|}
\hline Parameter & Symbol & Physical Units & Lattice Units \\
\hline \multicolumn{4}{|l|}{ Dimensional Parameters } \\
\hline Liquid density & $\rho_{l}$ & $0.996 \mathrm{~g} / \mathrm{cm}^{3}$ & 1.0 \\
\hline Gas density & $\rho_{g}$ & $0.0011925 \mathrm{~g} / \mathrm{cm}^{3}$ & $1.197 \times 10^{-3}$ \\
\hline Liquid dynamic viscosity & $\eta_{l}$ & $0.01 \mathrm{~g} /(\mathrm{cm} \cdot \mathrm{s}$ & $5.23 \times 10^{-3}$ \\
\hline Gas dynamic viscosity & $\eta_{g}$ & $1.835 \times 10^{-4} \mathrm{~g} /(\mathrm{cm} \cdot \mathrm{s}$ & $9.596 \times 10^{-5}$ \\
\hline Surface tension & $\sigma$ & $73.0 \mathrm{~g} / \mathrm{s}^{2}$ & 0.0318 \\
\hline Disk radius & $R$ & $0.16 \mathrm{~cm}$ & 100 \\
\hline Initial disk separation & $L_{0}$ & $0.32 \mathrm{~cm}$ & 200 \\
\hline Volume of liquid bridge & $V$ & $0.04 \mathrm{~cm}^{3}$ & $9.76 \times 10^{6}$ \\
\hline Velocity of upper disk & $U$ & $0.6 \mathrm{~cm} / \mathrm{s}$ & 0.0005 \\
\hline Gravity & $g$ & $981 \mathrm{~cm} / \mathrm{s}^{2}$ & $1.09 \times 10^{-6}$ \\
\hline \multicolumn{4}{|l|}{ Dimensionless Parameters } \\
\hline Viscosity ratio & $\eta_{l} / \eta_{g}$ & 54.5 & 54.5 \\
\hline Density ratio & $\rho_{l} / \rho_{g}$ & 835.2 & 835.2 \\
\hline Aspect ratio & $L_{0} / R$ & 2 & 2 \\
\hline Volume ratio & $V /\left(\pi R^{2} L_{0}\right)$ & 1.552 & 1.552 \\
\hline Bond number & $B o=\rho_{l} g R^{2} / \sigma$ & 0.3425 & 0.3425 \\
\hline Ohnesorge number & $O h=\eta_{l} / \sqrt{\rho_{l} \sigma R}$ & 0.002932 & 0.002932 \\
\hline Capillary number & $C a=\eta_{l} U / \sigma$ & $8.2197 \times 10^{-5}$ & $8.2197 \times 10^{-5}$ \\
\hline \multicolumn{4}{|l|}{ Numerical Parameters } \\
\hline Interface Thickness & $\delta$ & & 5 \\
\hline Mobility & $M$ & & 0.1 \\
\hline
\end{tabular}

Table 1: The parameters of the experiment and simulation. In the experiment, the liquid was water and the gas was air at $22^{\circ} \mathrm{C}$. 
as it does not significantly affect the physical behavior of the system.

Sunken and raised platforms were used to represent the disk to help with pinning of the contact line (Fig. 5). At first we specified a "fully wetting" contact angle $\left(5^{\circ}\right)$ over the disk area on a flat substrate and a fully non-wetting contact angle $\left(175^{\circ}\right)$ outside, but the fluid did not stay pinned properly due to gravitational effects. The fluid on the bottom disk overflowed the disk area and the fluid at the top disk did not fully cover the disk. The prescription of the sunken and raised platforms was a numerical convenience to pin the contact line where desired, as it was noticed that the contact line tends to get pinned at convex corners [35]. As a final note on boundary conditions, the boundaries at the side of the domain were held at constant pressure with a normal gradient free velocity.

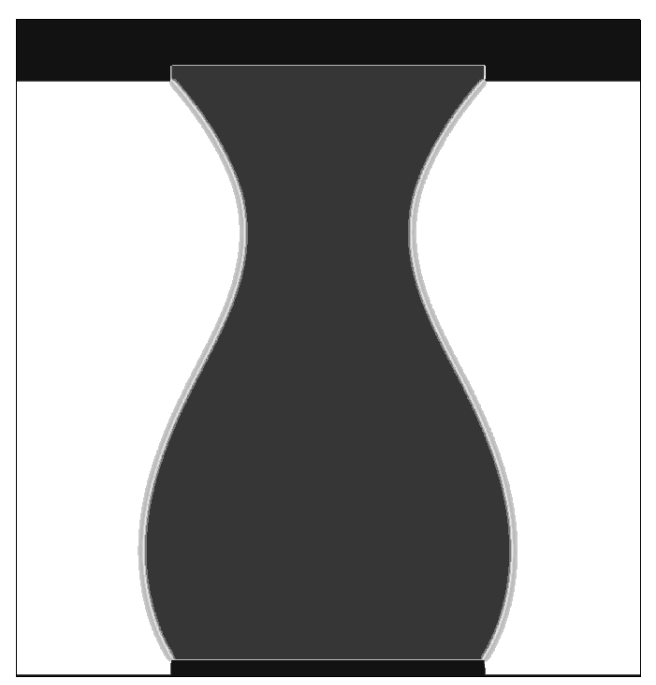

Figure 5: A 2D cross section of the simulation depicted in Fig. 4, more clearly showing the sunken and raised platforms. The presence of the platforms creates a convex "corner" that is a numerical aid to pin the bridge to the disk perimeter.

The minimum radius as a function of time is plotted for both the simulation and the experiment in Fig. 6. The simulation result compares favorably to the experiment, where the shape of the curve appears qualitatively correct, but with the dynamics proceeding with about a $10 \mathrm{~ms}$ delay. This could be a result of the discrete nature of the boundary conditions as mentioned above. The important point is that we were able to capture the rupture process both quantitatively and qualitatively. The minimum radius decreased slowly during the initial stage of separation where fluid oscillations are evident, but decreased quickly near rupture. An important characteristic of the rupture process is that all bridges display a self-similar character sufficiently close to rupture $[8,37,38]$. For viscous liquids during the final stage of rupture, the minimum radius should decrease with a slope linear in $\tau=t_{\text {breakup }}-t$ when measured on a log-log plot as in Fig. 7 , where 
time is interpreted as advancing from right to left.

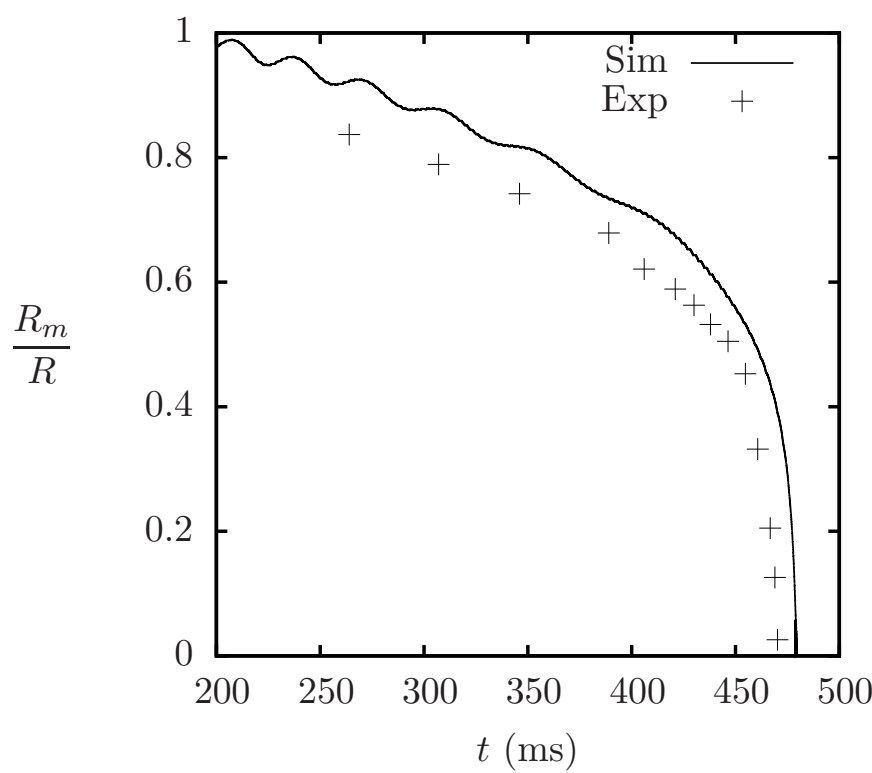

Figure 6: The minimum bridge radius normalized by the disk radius as a function of time, in milliseconds. The solid line is the simulation result and the symbols are the experimental result, digitized from [36].

Three other metrics of comparison are the normalized length at rupture, $L_{r} /\left.R\right|_{\text {sim }}=$ 3.80 and $L_{r} /\left.R\right|_{\exp }=3.75$ (based on Fig. 19 of [36]); the normalized axial position where rupture occurs, $l /\left.R\right|_{\operatorname{sim}}=2.32$ and $l_{r} /\left.R\right|_{\exp }=2.21$ (Fig. 6 of [36]); and the normalized volume of the sessile lower drop after rupture, $V_{l} /\left.V\right|_{\text {sim }}=0.783$ and $V_{l} /\left.V\right|_{\exp }=0.775$ (Fig.22 of [36]). Our simulation matches the experiment well according to these metrics which demonstrates that our simulations capture the salient points of stretching liquid bridges.

\section{Results}

In this section, an implementation of the method described above is used to simulate stretching liquid bridges containing solid particles. The analysis is separated into a discussion of fully wetting particles, where the contact angle is close to zero, and neutrally wetting particles, where the contact angle is $\theta=90^{\circ}$. We discuss the rupture length and pinch-off structure; the interface immersion effect on neutrally wetting particles; and the suppression of satellite drops and asymmetry of certain quantities after rupture. 


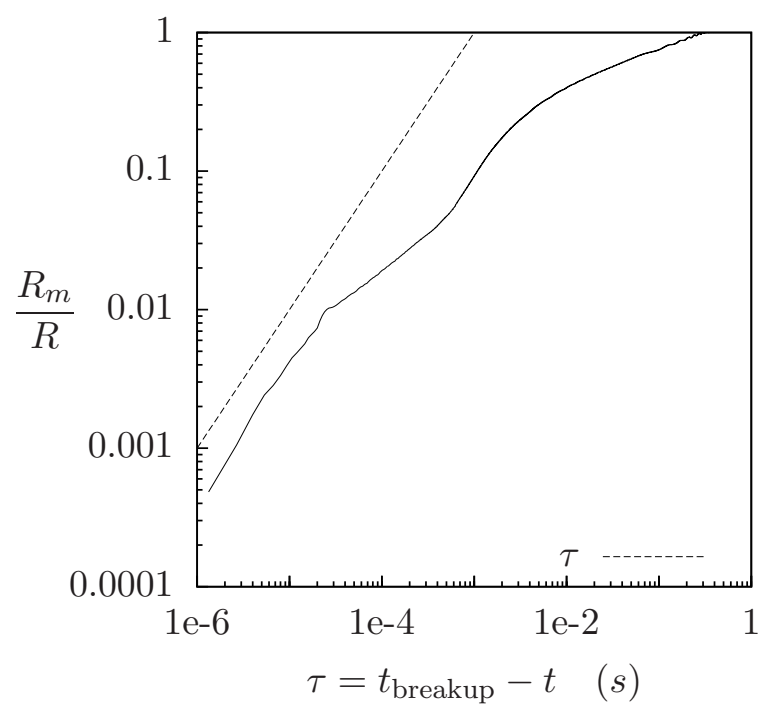

Figure 7: The minimum radius as a function of time before breakup, $\tau=t_{\text {breakup }}-t$ in seconds, on a log-log scale. Just before breakup, the radius should decrease proportional to $\tau$, as shown by the dashed line. 


\subsection{Rupture length and pinch off-structure}

It has been experimentally observed that the presence of fully wetting particles can delay rupture $[10-12,14]$. The prevailing idea is that for a suspension column large relative to the particle size, the suspension possesses an increased effective viscosity compared to the pure fluid, and this results in a greater resistance to (and thus slows) the thinning process. A typical effective viscosity model [39] is $\eta_{s}(\phi)=\eta\left(1-\phi / \phi_{\max }\right)$, where $\eta_{s}$ is the suspension viscosity, $\phi$ is the particle volume fraction, and $\phi_{\max }$ is an experimentally determined maximum packing fraction. This type of model explains the delay of rupture qualitatively, but does not account for the discrete nature of the particles. Thus it fails to capture aspects of the behavior when the effects of finite size become important, such as the structure at pinch-off.

We performed a set of simulations to analyze the effect of increasing particle volume fraction. The simulation setup was slightly different than Sec.3, as both walls were translated in opposite directions at speed $U$ to facilitate computations of quantities relative to the center of the bridge in the stretching direction. For simplicity, there were no platforms, and the desired wetted radius was maintained by using a fully wetting contact angle $\left(\theta=5^{\circ}\right)$ inside the disk region and a fully nonwetting contact angle $\left(\theta=175^{\circ}\right)$ outside.

The major difference between our simulations and existing experiments (including our validation studies) is that we did not incorporate effects of gravity in the simulations. Although most experimental studies of liquid bridge suspensions were driven by gravity forces as fluid was injected through a nozzle [10-12,14], we utilized stretching between parallel plates because it was more controlled from a numerical perspective. As we imposed a fixed extension rate of the bridge, we expect our results to have some differences when compared to experiments that allow for unconstrained bulk strain rate. Though actual rupture lengths may be different, the qualitative behavior of the systems is found to agree.

The simulations were initiated as a quiescent cylindrical column of fluid with particles dispersed at random. As the behavior was highly dependent on particle configuration, we performed a set of five simulations for each volume fraction considered, each with a random initial configuration, which enabled us to discern average trends and report standard deviation statistics. The walls were set in motion at initiation, and the simulation ran until a rupture was detected.

The parameters that define the simulation are the same as those of Sec.3 except for the addition of a particle radius $\left(R_{p}\right)$, particle density $\left(\rho_{p}\right)$, and the particle volume fraction $\left(\phi=V_{p} / V_{\text {tot }}\right)$, where $V_{p}$ is the volume occupied by the particles, and $V_{\text {tot }}$ is the total volume occupied by both fluid and particles. In this case, we removed the dimensionless Bond number(since there was no gravity), and replaced the Ohnesorge number with the more convenient Reynolds number $\left(R e=\rho_{l} U R / \mu_{l}\right)$. We chose to simulate a system with large fluid-fluid density and viscosity contrasts (similar to a water-air system). 
Given the large disparity between liquid and gas properties, the important parameters are the Reynolds and capillary numbers, which are typical for existing experiments. The parameters that define the system are listed in Table 2.

\begin{tabular}{lcl} 
Parameter & Symbol & Lattice Units \\
\hline Simulation Parameters & & \\
\hline Liquid density & $\rho_{l}$ & 1.0 \\
Gas density & $\rho_{g}$ & $1.188 \times 10^{-3}$ \\
Particle density & $\rho_{p}$ & 1.0 \\
Liquid dynamic viscosity & $\eta_{l}$ & 0.5 \\
Gas dynamic viscosity & $\eta_{g}$ & $9.75 \times 10^{-3}$ \\
Surface tension & $\sigma$ & 0.01 \\
Disk radius & $R$ & 50 \\
Particle radius & $R_{p}$ & 5 \\
Initial disk separation & $L_{0}$ & 100 \\
Volume of liquid bridge & $V$ & $7.85 \times 10^{6}$ \\
Disk velocity & $|U|$ & 0.001 \\
Interface Thickness & $\delta$ & 5 \\
Mobility & $M$ & 0.1 \\
\hline Dimensionless Parameters & & \\
\hline Viscosity ratio & $\eta_{l} / \eta_{g}$ & 51.3 \\
Liquid-Gas density ratio & $\rho_{l} / \rho_{g}$ & 842 \\
Liquid-Particle density ratio & $\rho_{l} / \rho_{p}$ & 1 \\
Aspect ratio & $L_{0} / R$ & 2 \\
Particle-disk size ratio & $R_{p} / R$ & 0.1 \\
Volume ratio & $V /\left(\pi R^{2} L_{0}\right)$ & 1.0 \\
Reynolds number & $R e=\rho_{l} U R / \eta_{l}$ & 0.1 \\
Capillary number & $C a=\eta_{l} U / \sigma$ & 0.05 \\
\hline
\end{tabular}

Table 2: The parameters of the simulations.

As in experiments (Fig. 8), increasing the volume fraction of particles increased the rupture length of the suspension bridges relative to the particle-free case, for both fully wetting and neutrally wetting particles (Fig. 9). However, rupture for neutrally wetting particles was accelerated compared to the fully wetting case for all volume fractions. Snapshots from particular volume fraction trials are shown in Fig. $10\left(\theta=5^{\circ}\right)$ and Fig. $11\left(\theta=90^{\circ}\right)$ at $t=5 \times 10^{4}$, when the length of the liquid bridge was twice its initial length. The increasing rupture length as $\phi$ increases seen in Fig. 9 supports the effective viscosity description, but the variability of the different trials shows that the discreteness of the particles plays an important role.

Fig. 12 shows a comparison of the pinch-off structure of a $\phi=0.30$ suspension of fully 


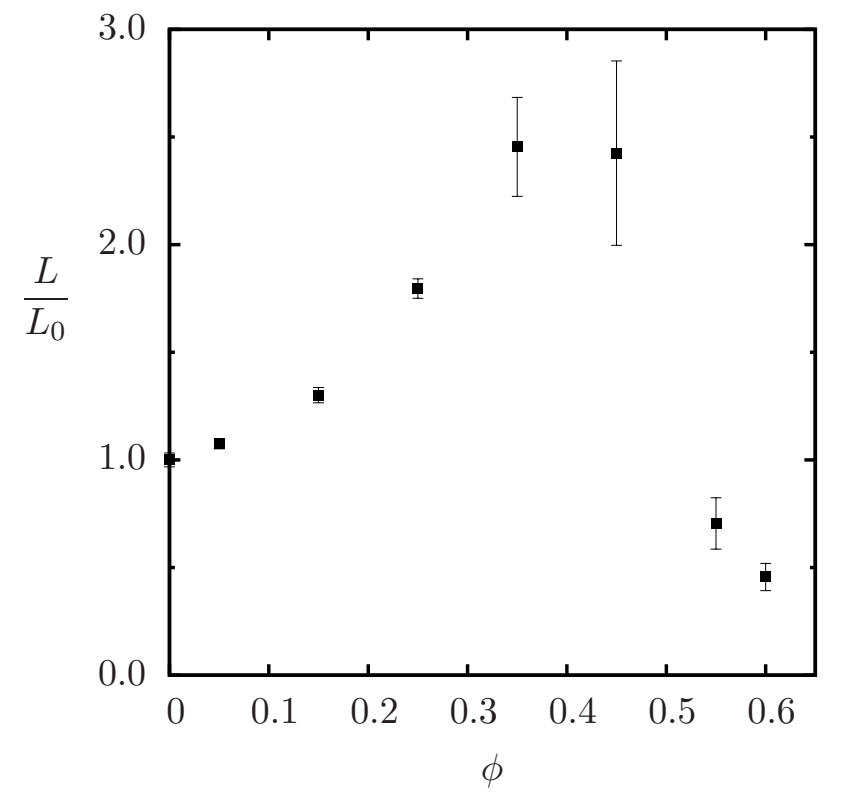

Figure 8: The rupture length, $L$, normalized by the particle-free rupture length, $L_{0}$, as a function of volume fraction $(\phi)$ for an experiment with fully wetting particles. The experiment was able to probe larger volume fractions than the simulations, identifying a volume fraction where the rupture length no longer increased and eventually became less than in the particle-free. The interval of volume fraction covered by our simulations produced rupture lengths that increased monotonically with volume fraction. 


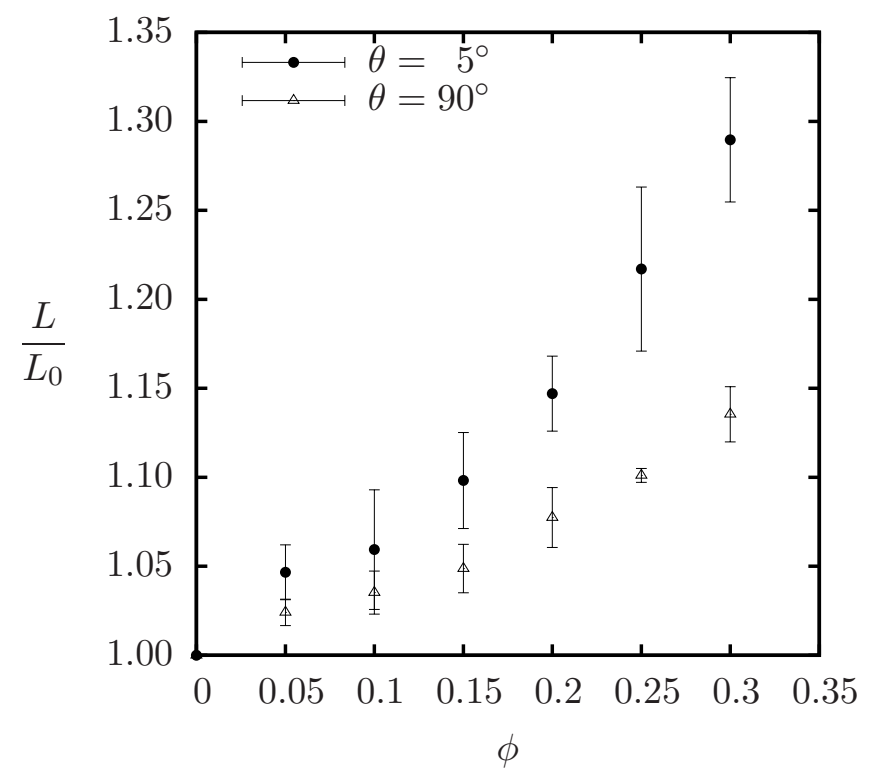

Figure 9: The rupture length, $L$, normalized by the particle-free rupture length, $L_{0}$, as a function of volume fraction $(\phi)$ for fully wetting particles $\left(\theta=5^{\circ}\right)$ and neutrally wetting particles $\left(\theta=90^{\circ}\right)$.

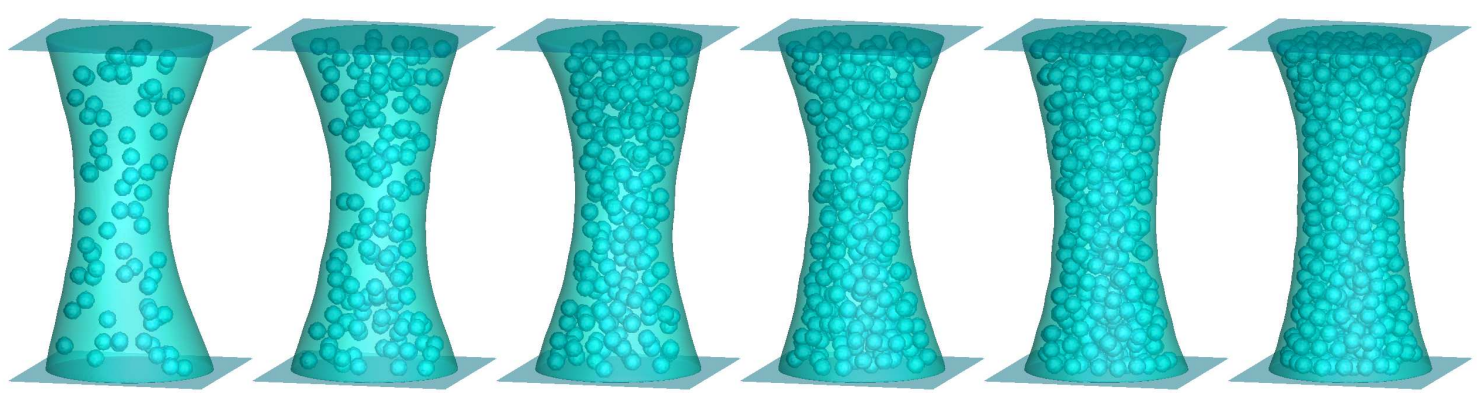

Figure 10: Snapshots of representative trial simulations for each volume fraction of fully wetting particles. The snapshots were taken at $t=50,000$ time steps, when the length of the liquid bridge was twice its initial length. The particle volume fractions from left to right are $\phi=0.05,0.10,0.15,0.20,0.25$, and 0.30 , respectively. 


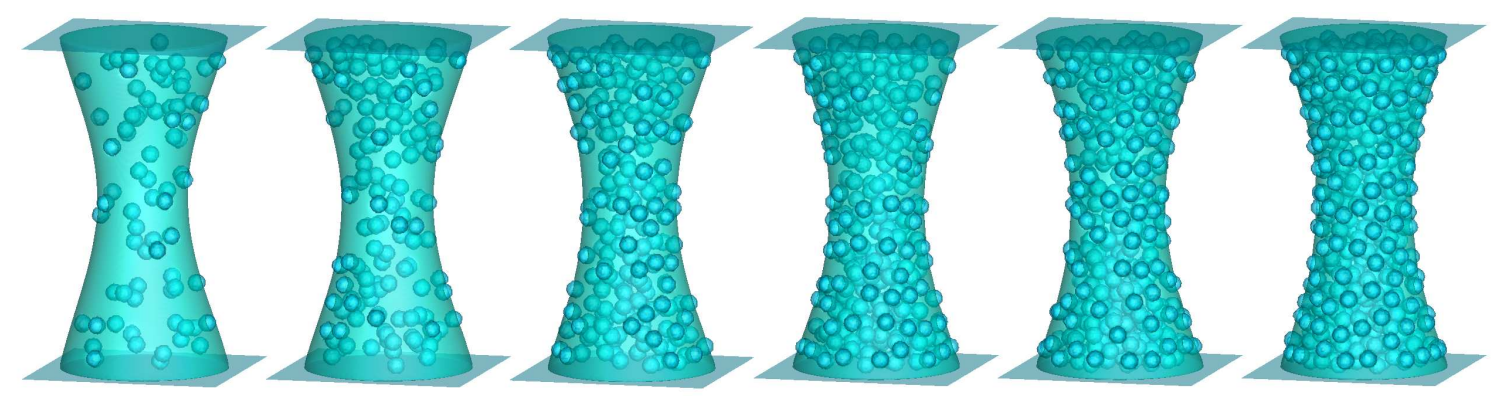

Figure 11: Snapshots of representative trial simulations for each volume fraction of neutrally wetting particles. The snapshots were taken at $t=50,000$ time steps, when the length of the liquid bridge was twice its initial length. The particle volume fractions from left to right are $\phi=0.05,0.10,0.15,0.20,0.25$, and 0.30 , respectively.

wetting particles and a pure fluid with viscosity equal to the effective viscosity of that suspension. The pure fluid with effective viscosity does not match the rupture length (does not fall within the standard deviation) or pinch-off structure. The presence of rigid particles is ultimately responsible for such a shape, which is not predicted by an effective viscosity model. Typical pinch-off structures for different volume fractions, $\phi$ are displayed in Fig. $13\left(\theta=5^{\circ}\right)$ and Fig. $14\left(\theta=90^{\circ}\right)$.

For fully wetting particles, there is a fundamental difference in behavior between small $(\phi \leq 0.10)$ and large volume fraction simulations. As has been suggested previously $[10,11]$, particles in the central portion of the bridge, or throat, region of low volume fraction bridges can freely follow the fluid as it flows out of that region. For large volume fraction simulations, particle motion is inhibited by the presence of other particles. This is illustrated by a sequence of snapshots for typical small and large $\phi$ simulations in Figs. $15 \mathrm{a}$ and $\mathrm{b}$, respectively. The effect of particles at the early stage delayed rupture in a manner similar to an effective viscosity, which is the main reason why the rupture length increased with volume fraction. Rupture did not occur, for either case, until a relatively particle-free region was sampled in the later stage. The variability in the results clearly indicates that the discreteness of the particles determined when and where this particlefree region developed.

For small volume fractions, most particles were able to escape the throat region before the minimum radius necked down to the size of the particles. This resulted in a rupture structure that closely resembled the particle-free case, the so-called needle-and-sphere configuration [11], where nearly spherical pendant and sessile drops are connected by a needle-like liquid thread. As there were more particles in the $\phi=0.10$ case, a few sometimes stayed near the throat delaying rupture, which resulted in a larger standard deviation of the rupture length.

As the volume fraction increased, the particle concentration became more uniform which decreased fluctuations in rupture length $(0.15 \leq \phi \leq 0.20)$. However, for the 


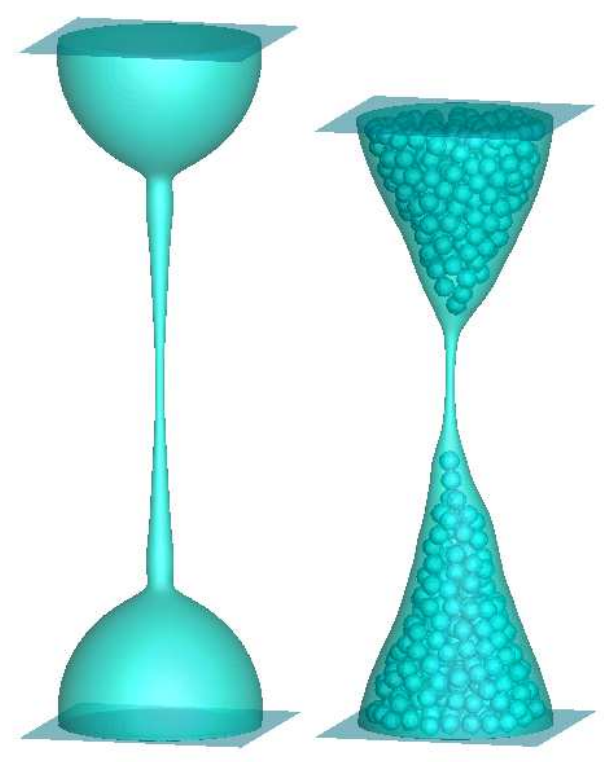

Figure 12: A comparison of the pinch-off structure for a pure fluid with viscosity equal to the effective viscosity of a $\phi=0.30$ suspension and the corresponding suspension, assuming the model of reference [39] with $\phi_{\max }=0.64$. The bottom substrates are aligned to clearly illustrate the difference in rupture length.

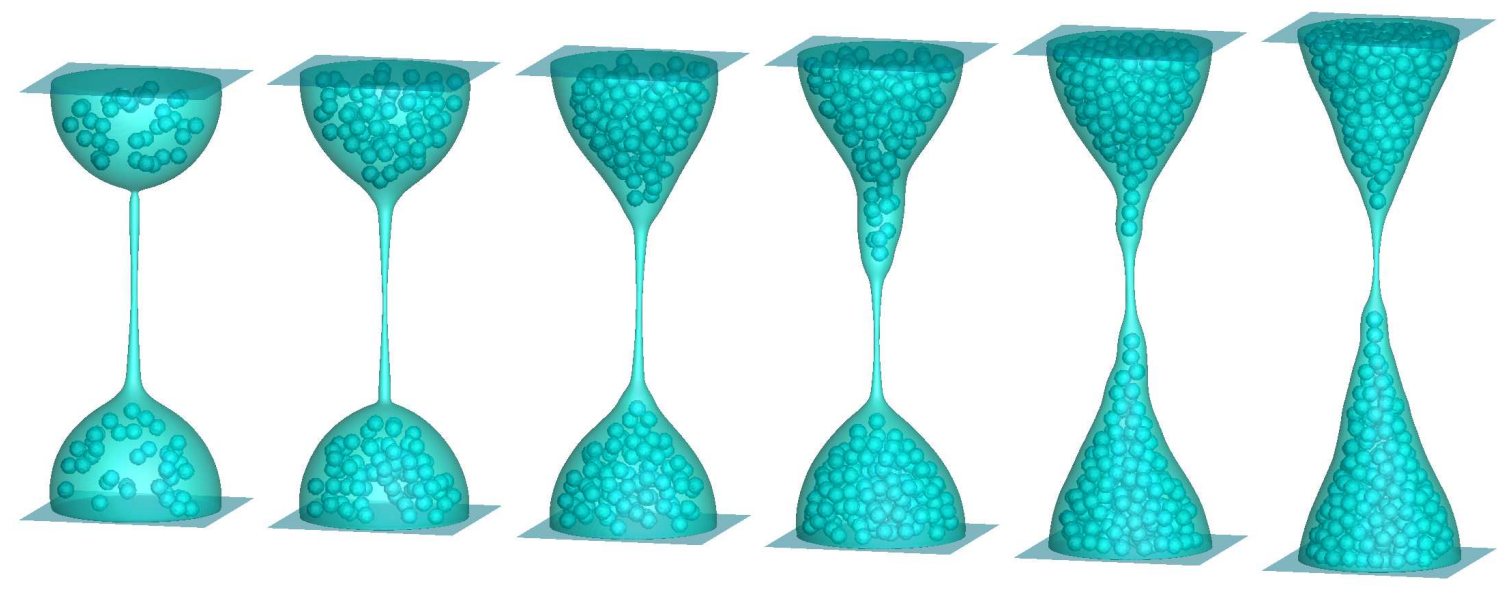

Figure 13: Snapshots of representative trial simulations for each particle volume fraction of fully wetting particles just before rupture. The volume fractions from left to right of the top row are $\phi=0.05,0.10,0.15,0.20,0.25$, and 0.30 , respectively. 


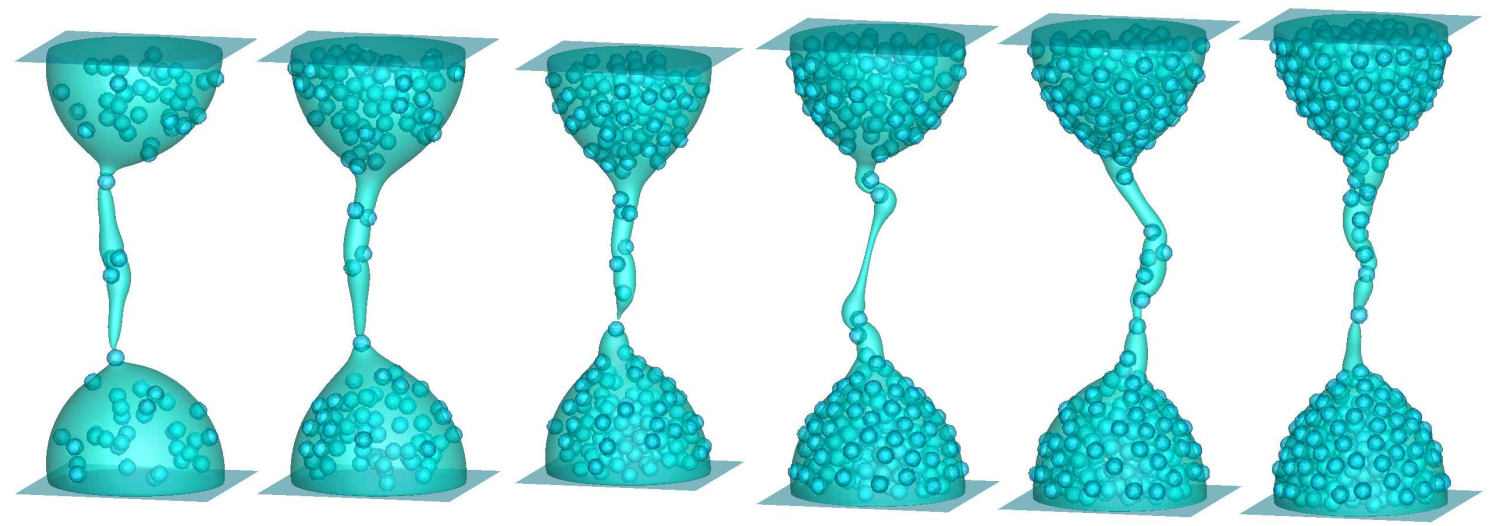

Figure 14: Snapshots of representative trial simulations for each particle volume fraction of neutrally wetting particles just before rupture. The volume fractions from left to right of the top row are $\phi=0.05,0.10,0.15,0.20,0.25$, and 0.30 , respectively.
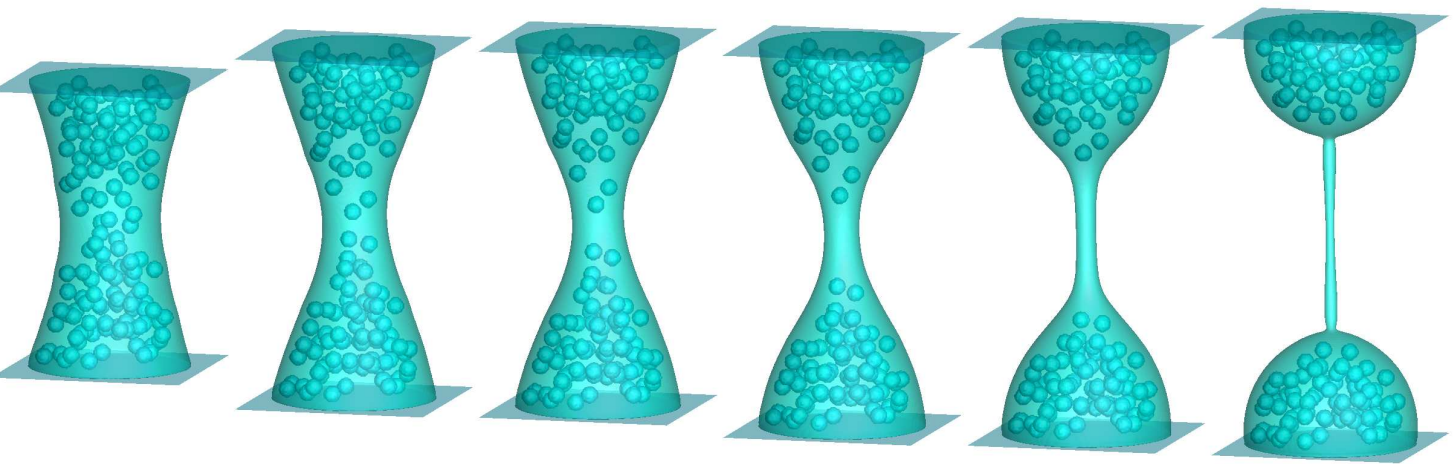

a)
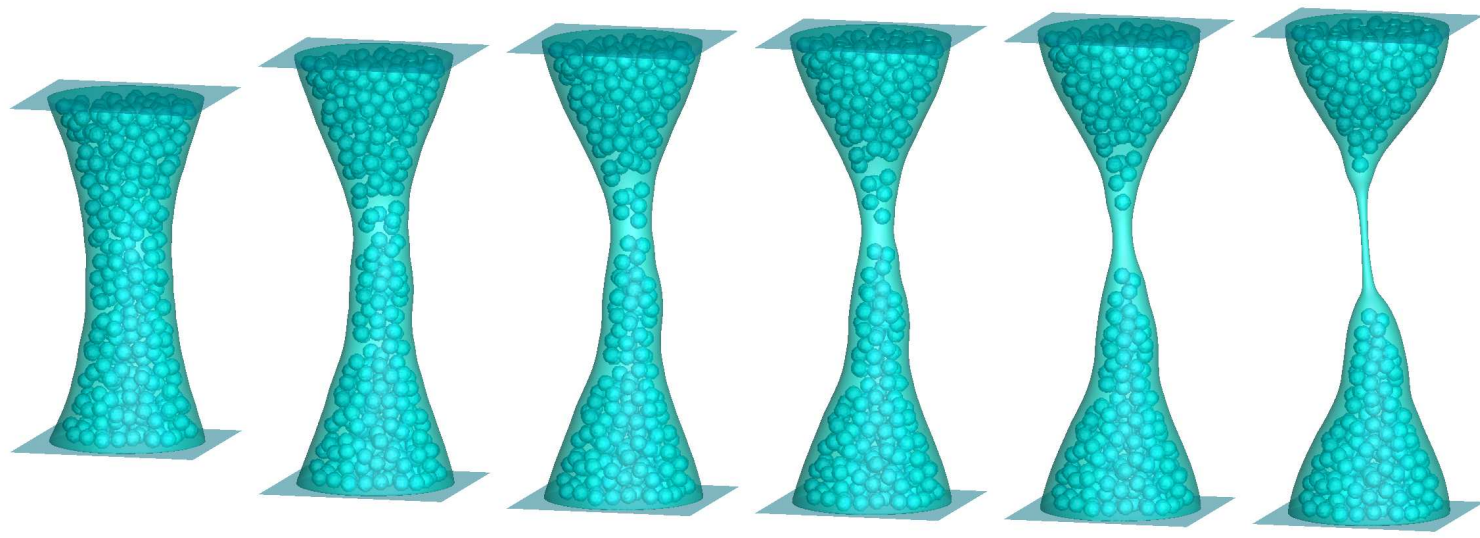

b)

Figure 15: Time sequences of characteristic simulations of fully wetting particles with volume fraction a) $\phi=0.10$ and b) $\phi=0.25$. 
largest volume fractions, $\phi \geq 0.25$, the development of a particle-free region became highly configuration dependent, as evidenced by an increased standard deviation of rupture length. Furthermore, the effect of large volume fraction can be seen in the pinch-off structure, where clusters of particles retreating back into the drop formed a tail as the individual particle motion was inhibited by other particles. For the largest volume fractions, a major departure from spherically shaped drops occurred as the suspension adopted a conical shape. For all of these structures, a dynamical feature held in common was that a particle-free liquid thread was sampled just before rupture, where the length of the thread was inhibited from growing when it was terminated by a particle.

A set of experiments was run to qualitatively corroborate the general findings. As mentioned previously, the experiments were run by injecting the suspension through a nozzle, and fell under gravity, but with ultimate rupture driven by capillary forces. As the size of particles used in the experiments could not feasibly be matched in the simulations, one can not expect quantitative agreement. Specifically, experimental datasets were produced by slowly extruding suspensions with varying volume fractions of $33 \mu \mathrm{m}$ polyethylene particles. The particles had a density of $1 \mathrm{~g} / \mathrm{mL}$ and were suspended in 20 cst silicone oil (Sigma Aldrich AR20) of the same density. In all of the experiments, the nozzle was $4.7 \mathrm{~mm}$ in diameter. The suspension was extruded at a constant rate by a syringe pump (Razel Scientific R99-E) at a flow rate of $10^{-4} \frac{\mathrm{mL}}{\mathrm{s}}$, making the extrusion process effectively quasi-static. All experiments were performed in a temperatureand humidity-controlled laboratory $\left(50 \pm 5 \%\right.$ humidity and at $\left.22 \pm 0.5^{\circ} \mathrm{C}\right)$. The breakup process was captured with a fast camera typically capturing 1000-7000 frames per second.

Comparing sequences of images of the detaching droplet (Fig.16) to the simulation results, we found qualitatively similar structures: pure liquid-like breakups occur at low volume fractions and symmetric double cones emerge at higher densities. Quantitatively, however, we found the experiments transitioned to the structures associated with suspensions (as opposed to remaining close to the forms seen in pure liquids) at higher volume fractions.

\subsection{Interface immersion effect on neutrally-wetting particles}

Neutrally wetting particles behave as do fully wetting particles when they remain in a bulk fluid region, and such particles also increase the rupture length, consistent with an effective viscosity argument. However, a different mechanism is seen when particles become immersed in the interface, and become subject to unbalanced capillary forces tangent to the interface.

Due to the deformation of the liquid bridge, the interface approaches immersed particles. If the intervening fluid between the particle and interface drains away, nucleation of a liquid-gas-solid contact line can occur [40]. At nucleation, the contact line is not at the equilibrium contact angle, so it will spread over the particle. As the contact line spreads, capillary forces normal to the liquid bridge surface will pull the particle into the 

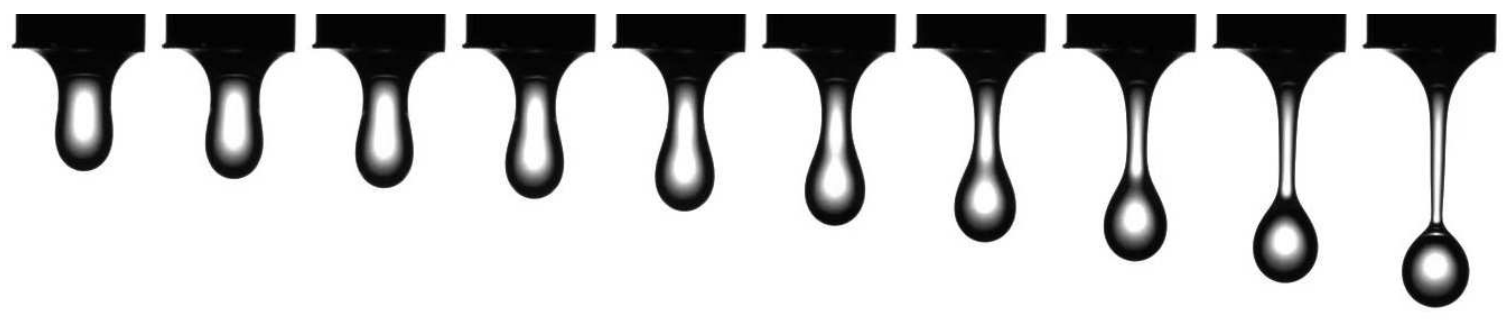

(a) $\phi=0.00$
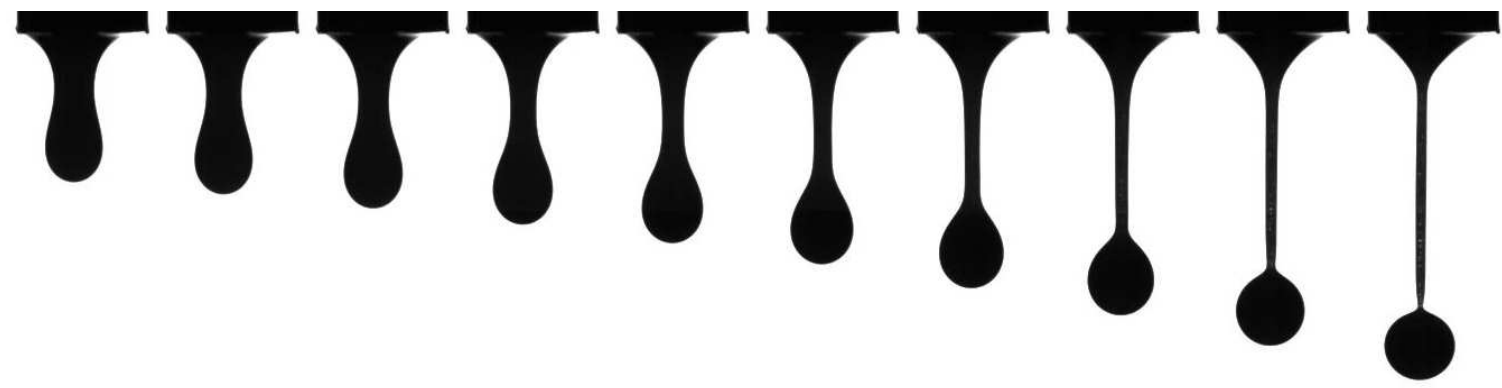

(b) $\phi=0.15$
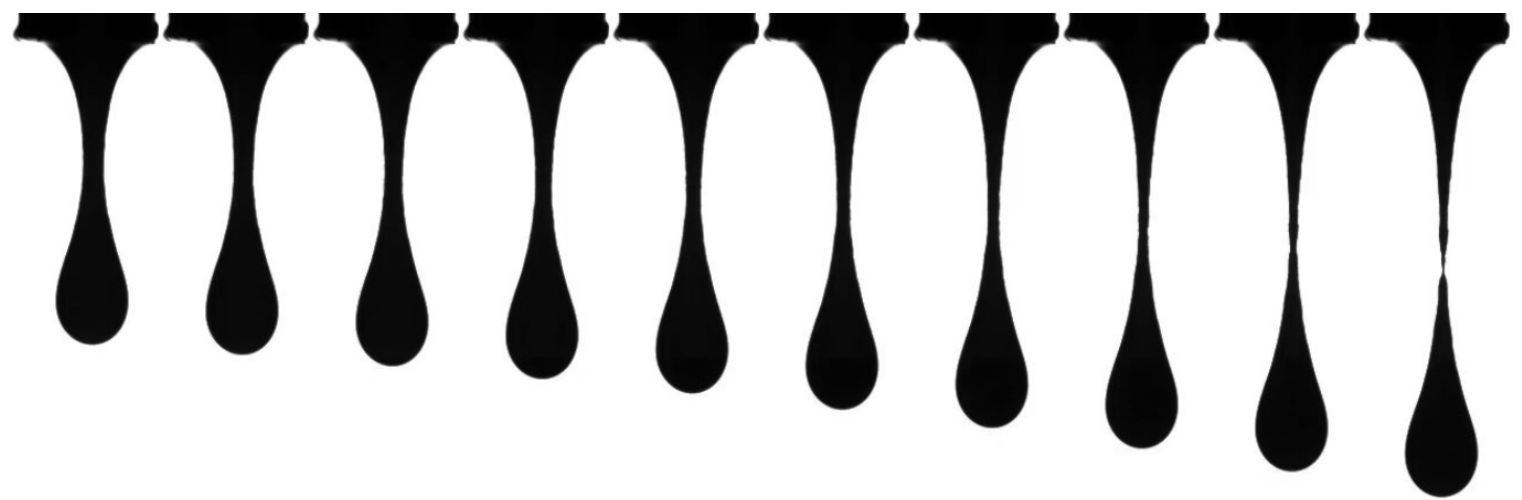

(c) $\phi=0.55$

Figure 16: Characteristic break-up structures. Time runs from left to right and images (spaced by roughly $0.03 \mathrm{~s}$ ) are synced at the breakup instant. For low volume fractions, the inclusion of particles tends to increase the length of detaching suspensions until $\phi \approx 0.40$, above which the neck becomes appreciably shorter and more symmetric. At $\phi=0.15$, the breakup structure is similar to the particle-free case $(\phi=0.0)$ At $\phi=0.55$, the breakup resembles the conical structure seen in the higher- $\phi$ simulations and prior experiments [10]. 
interface. The combination of particle and contact line motion will stably immerse, or embed, the particle in the interface.

The immersion of a particle in the interface is an example of an event that is a computational singularity, similar to other events like drop coalescence or rupture, where distinct topologies are either combined or separated. An advantage of our diffuse interface scheme is that it can capture these events without intervention. In contrast to this, sharp interface methods must explicitly change their topology, based on some criterion, in order for such events to take place. The disadvantage of diffuse interface methods is that the singular event, in our case particle nucleation of a three phase contact line, may occur before the expected completion of the drainage process. For small particles, however, the drainage process takes place over a timescale equal to that of the expansion of the contact line [40], which is a fast process compared to the large scale dynamics of the simulation. So while the immersion of particles into the interface probably occurs prematurely, the bulk dynamics can be expected to be relatively accurately reflected.

Once in the interface, unbalanced capillary forces tangent to the interface drive the neutrally-wetting particles toward the throat region, or at least prevent them from being evacuated as quickly as interior particles [42]. This is illustrated by a sequence of snapshots for typical small and large $\phi$ simulations in Figs. 17a and b, respectively. The capillary forces arise from differences in interface curvature along the contact line. Particles near the throat region sample a larger variation of interface curvature than particles close to the end disks, and therefore experience a larger capillary force. The consequence is that particles are present in the thread, preventing further thinning and accelerating rupture compared to the fully wetting case.

\subsection{Satellite drop suppression and post-rupture asymmetry}

As seen in experiments [10], a consequence of including fully wetting particles is the suppression of satellite drops. For the particle-free case (Fig. 18a), rupture of the thread occurred nearly simultaneously at the connection to the pendant and sessile drops, forming one satellite drop that eventually ruptured again at the center into two satellite drops. When a small number of fully wetting particles are added (Fig.18b), the rupture structure is altered, diminishing the number of satellite drops. The behavior is highly configuration dependent for small volume fractions, resulting in a large standard deviation in the number of satellite drops. As the volume fraction is increased, the behavior no longer differs from the particle-free case, and the resulting small particle-free threads rupture in their center and do not form satellite drops. Since neutrally wetting particles are not evacuated from the thread prior to rupture, they tend to promote the formation of satellite drops for all volume fractions (Fig. 18b), where some satellite drops contain particles as well (c.f. Fig. 15).

We note one further consequence of including particles, namely the introduction of asymmetry. Consider the possible application depicted in Fig. 19 where a nozzle extrudes 


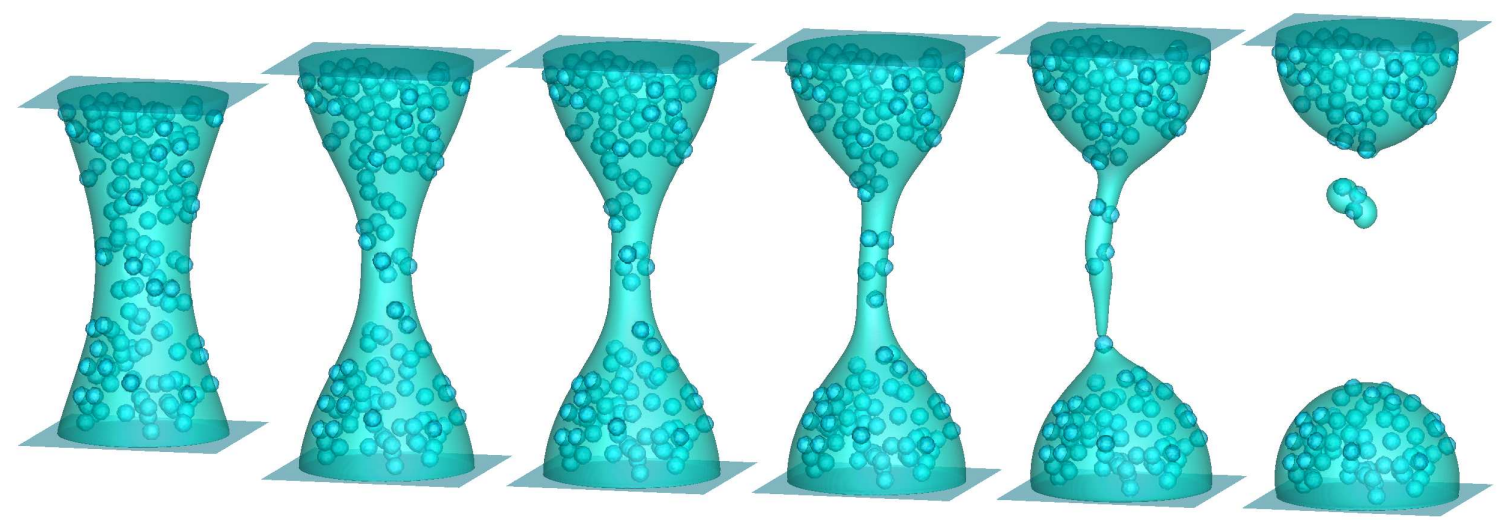

a)

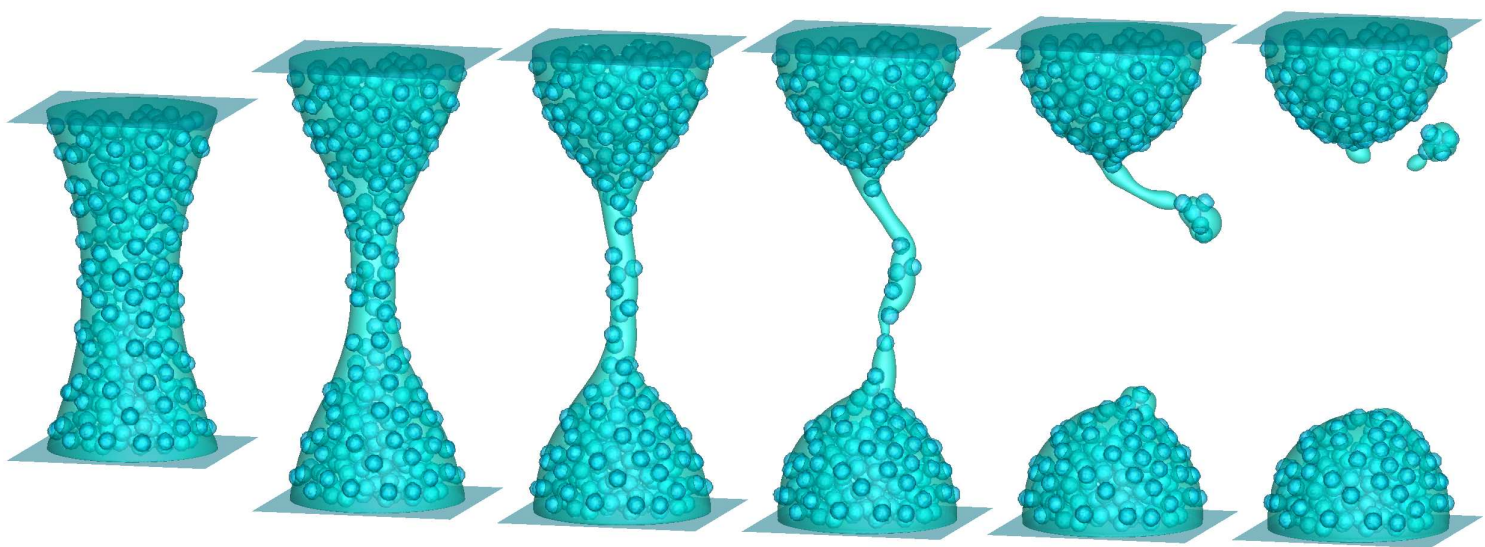

b)

Figure 17: Time sequences of characteristic simulation of neutrally wetting particles with volume fraction a) $\phi=0.10$ and b) $\phi=0.25$. 


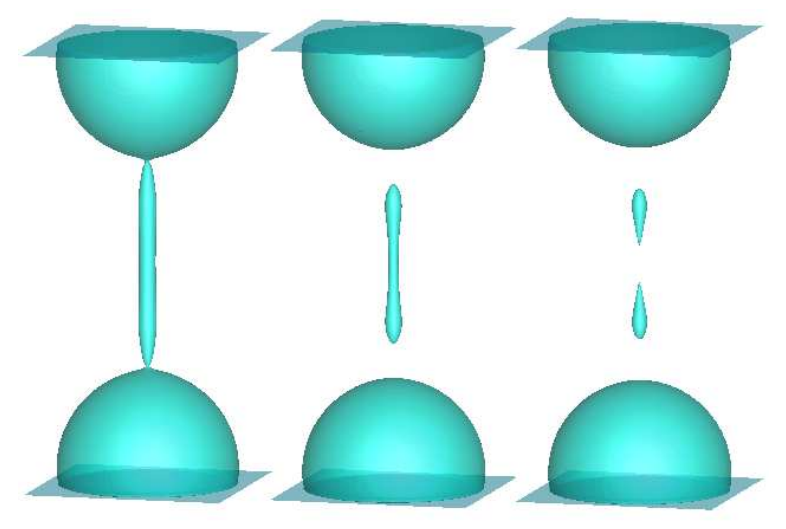

(a) The rupture sequence for the particle-free case. Pinch-off first occurred at the connection of the thread to the pendant and sessile drops nearly simultaneously, forming a single satellite drop which subsequently ruptured again to form two drops.

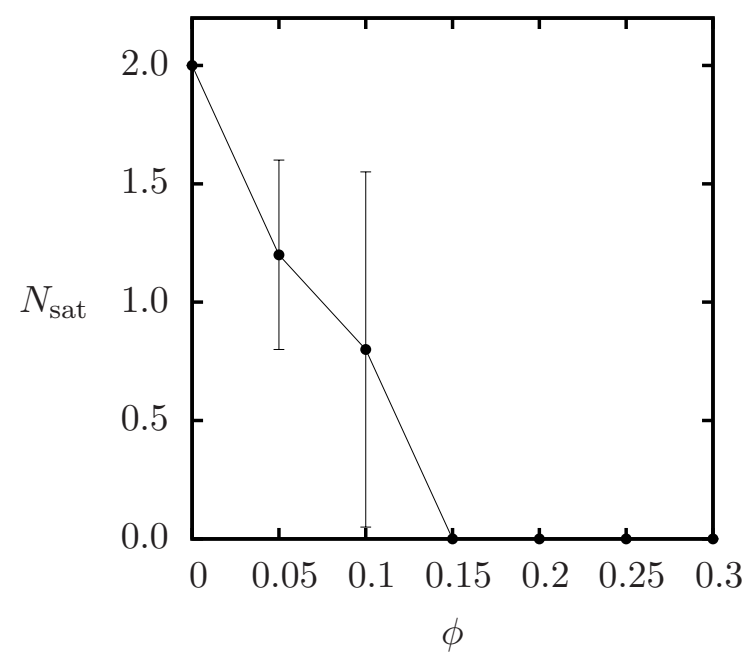

(b) The average number of satellite drops as a function of $\phi$ for $\theta=5^{\circ}$. The inclusion of fully wetting particles suppressed satellite drops. For small $\phi$, there were still some satellite drops created, but it highly depended on the configuration, as seen by the large standard deviation.

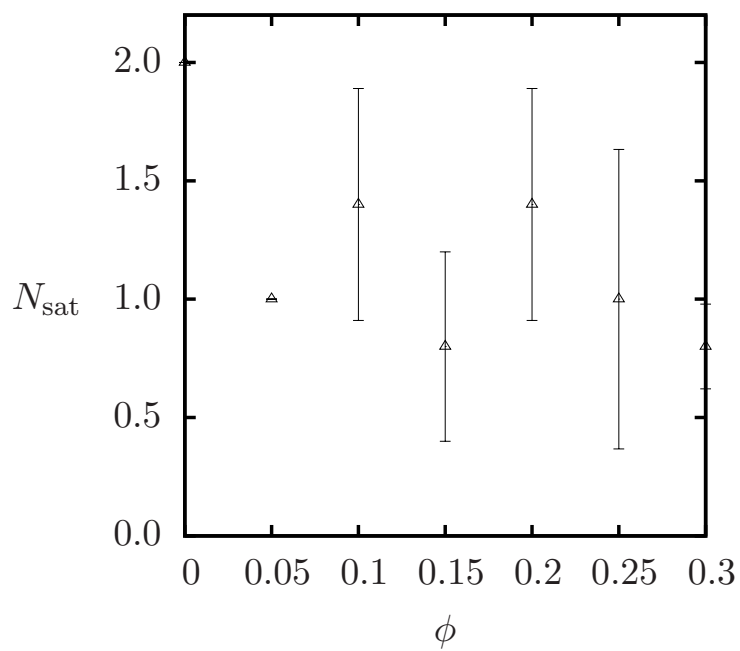

(c) The average number of satellite drops as a function of $\phi$ for $\theta=90^{\circ}$. The inclusion of neutrally wetting particles promoted the generation of satellite drops, some containing particles themselves, for all $\phi$.

Figure 18: (a) An image sequence of satellite drops formed in the particle-free case and the average number of satellite drops (with standard deviation) as a function of $\phi$ for (b) fully wetting particles, and (c) neutrally wetting particles. 
a volume of suspension to be deposited on a substrate in a desired amount. The nozzle is brought in contact with the substrate where the suspension wets a specified area. As the nozzle is retracted, the bridge ruptures leaving suspension behind. Depending on the particle configuration, the axial location of rupture can vary widely, leading to asymmetry in the volume of pendant and sessile drops and number of particles contained in those drops. For printing applications, such potential variability in deposited partial volume and concentration of particles should be considered.
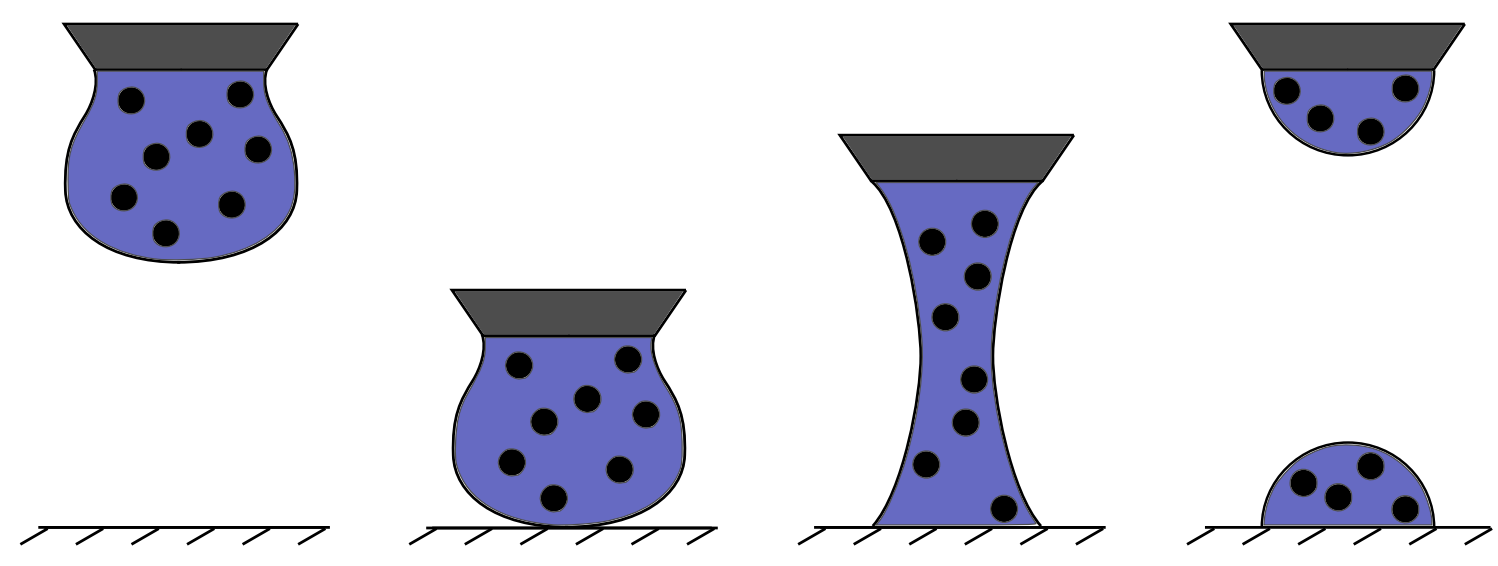

Figure 19: Schematic of a deposition application where suspension material extruded from a nozzle is brought into contact with a substrate to deposit a desired amount. The nozzle is retracted, stretching the bridge to rupture. If there were no particles, they resulting pendant and sessile drops would be expected to be nearly identical, but the presence of particles introduces asymmetry and some uncertainty into the rupture process.

In Fig. 20a is a plot of the axial location of rupture as a function of solid fraction for fully wetting particles. Since the rupture location $\left(l_{R} \in[0: L]\right)$ could be to either side of the axial midpoint $(L / 2)$, depending on configuration, an appropriate measure of rupture asymmetry is the magnitude of the displacement from the axial midpoint. For $\phi=0.05$, the behavior was highly configuration dependent as there was a large deviation about the mean, meaning the rupture either occurred far from the center, as in the particle-free case, or particles lingering in the throat caused the rupture to occur near the axial midpoint $\left(l_{R}-L / 2 \approx 0\right)$. This limits the predictability of rupture location for this volume fraction. A predictable result is interpreted as occurring near the axial midpoint with little deviation. The further away from the axial midpoint, the wider the effective standard deviation, as it is not possible, without gravity, to predict which side of the midpoint rupture will occur. Increasing the volume fraction to $\phi=0.10$ focuses the rupture location much more toward the center of the bridge. Further increase in the volume fraction results in a monotonic increase in the departure from the midpoint for rupture. For neutrally wetting particles (Fig.20a), rupture occurs much more regularly away from the axial midpoint. The partial volumes of the two masses left after rupture 


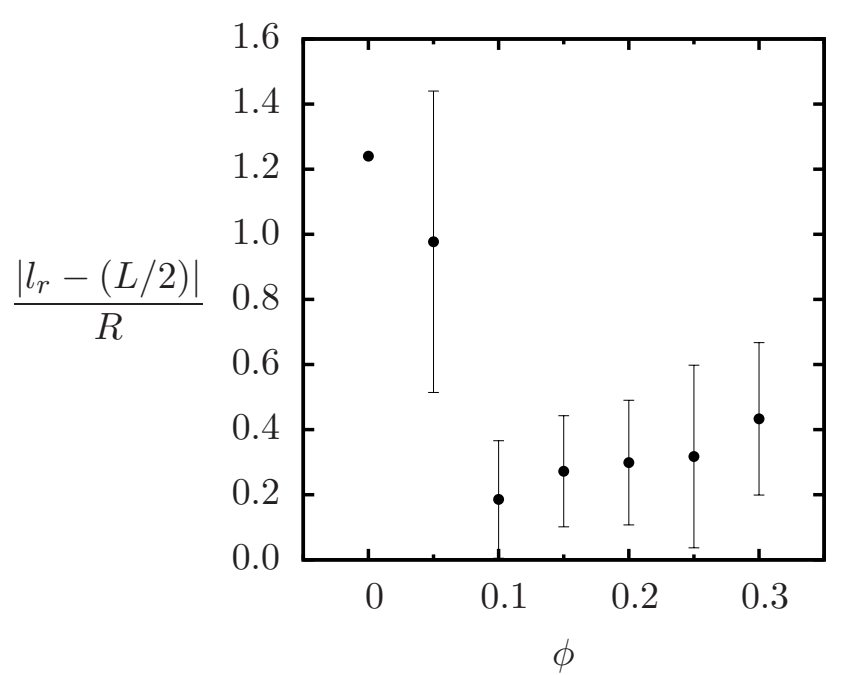

(a) Fully wetting particles.

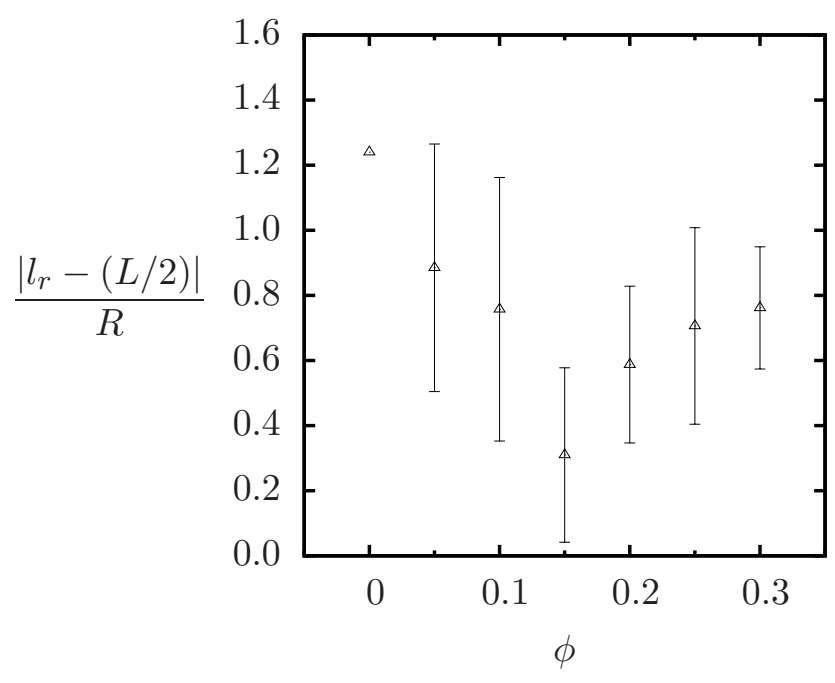

(b) Neutrally wetting particles.

Figure 20: The average rupture location, relative to the bridge center at $L / 2$, normalized by the bridge radius, as a function of $\phi$.

vary much less than the rupture length indicating the asymmetry is predominantly in the thread which is of small volume. The variance in volume is less for the neutrally wetting case, at about $2-4 \%$, while for fully wetting particles the variability was $7-12 \%$, for $\phi>0.1$. Both wetting conditions have about $4 \%$ variance at $\phi=0.05$. Our simulations thus indicate that deposited drops at higher $\phi$ would be more regular when using neutrally wetting particles. However, it should also be kept in mind that neutrally wetting particles generate more satellite drops.

Related to the asymmetry in volume, there is some asymmetry in the number of particles in the two masses after rupture, and this is found for fully wetting particles to be near $10 \%$ at each $\phi$, as shown in Fig. 21a. The asymmetry in number of neutrally wetting particles decreases with increasing $\phi$, and exhibits less deviation.

\section{Conclusions}

We have adapted our previously developed three-phase (liquid-gas-solid particle) latticeBoltzmann method (LBM) to describe stretching of particle-laden liquid bridges. The liquid bridges were stretched by separating two parallel plates at a fixed velocity. The implementation of the method was validated by comparing the minimal bridge radius as a function of time to a particle-free experiment. Although this mode of stretching is 


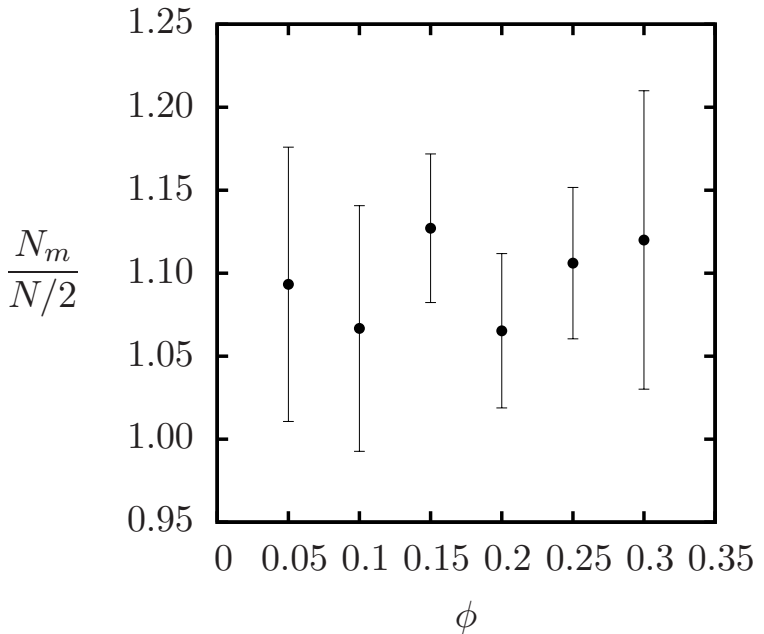

(a) Fully wetting particles.

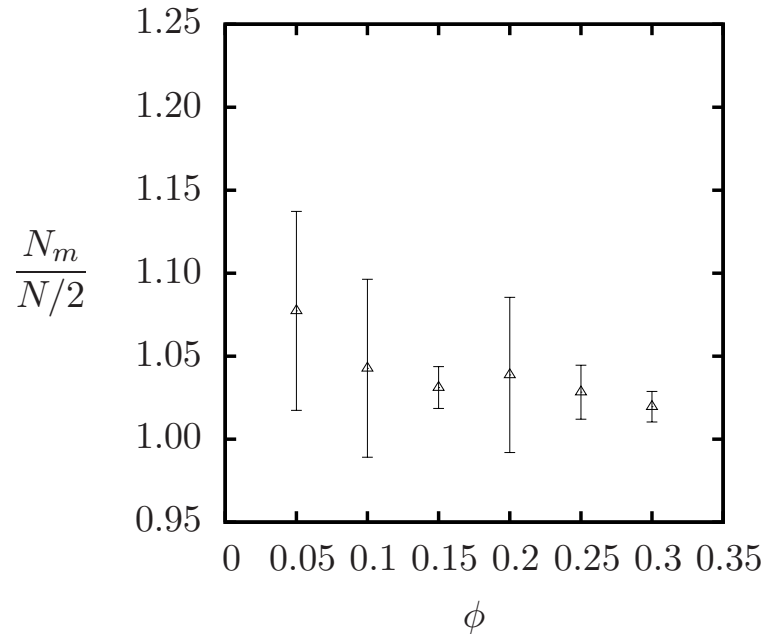

(b) Neutrally wetting particles.

Figure 21: The maximum post-rupture number of particles (the greater number contained in either the sessile or pendant drop) normalized by half the total number of particles as a function of $\phi$. 
different from most experimental work where particle-laden drops issue from a nozzle, a comparison to experiments on extruded drops finds similar basic features.

The study focused on a rupture comparison between liquid bridges that contained fully wetting (FW) particles, as in most experiments, and neutrally wetting (NW) particles, as a function of particle volume fraction, $\phi . \mathrm{FW}$ particles increased the rupture length with increasing volume fraction relative to the particle-free case, as seen in experiments. NW particles also increased the rupture length, but less so. A possible explanation is that NW particles can enter the interface, where they become susceptible to capillary forces. These capillary forces tend to drive the particles toward the throat, or at least slow down their escape from that region, causing an earlier initiation of the final stage of rupture, where particles directly interact with the thread.

The rupture structure was also analyzed, where it was first demonstrated that the pinch-off structure for a particle-laden liquid bridge was different from the structure of a particle-free liquid bridge with the effective viscosity of the suspension, as seen in experiments. For FW particles, the structure at low volume fractions was very similar to the particle-free case as particles were free to escape the throat region near rupture. As the volume fraction increased, tails at pinch-off became evident as particle motion escaping the throat was inhibited by other particles. A transition to a conical structure occurred for the largest volume fractions. However, a thin particle-free thread was observed just prior to rupture for all $\phi$. As NW particles were able to enter the interface, they presented less of a barrier to their neighbors, and the pinch-off structure resembled the particle-free needle-and-sphere shape for all but the largest volume fractions.

Upon rupture, bridges with FW particles tended to suppress satellite drops. As $\phi$ increased, the length of the filament of interstitial fluid connecting the pendant and sessile suspension drops decreased and tended to rupture in its center, inhibiting the production of satellite drops. Thus, our calculations have provided insight to the experimentally observed behavior [10]. On the other hand, bridges with NW particles maintained the particle-free structure where there was a long needle-like thread which tended to produce satellite drops. Therefore, there was no noticeable suppression of satellite drops for any $\phi$. Furthermore, as a consequence of the increased wettability and the tendency to remain in the rupture region, NW particles were also present in the satellite drops.

Another consequence of the pinch-off structure was the asymmetry of certain variables after rupture. While numerical artifacts may play a role in promoting asymmetry, the driving force behind it is the asymmetry created by the particles. For FW particles, the bridge ruptured far from the center of the bridge (as in the pure liquid case) for only the smallest $\phi$ studied. The bridge ruptured away from the center for all volume fractions of NW particles. The volumes of the pendant and sessile drops were erratic for FW particles, but surprisingly regular for NW particles. The variability for FW particles was a result of a larger amount of suspended solid being present in the rupture zone, carrying with it a large change in the two volumes formed by asymmetric rupture. Since the bulk of the fluid was already in the pendant/sessile drops for NW particles, there 
was relatively little suspension in the thread, and any asymmetry in rupture implied only a small change in the drop volumes. Finally, there was no noticeable difference in the number of FW particles present in the pendant/sessile drops after rupture as a function of volume fraction, but the number of NW particles became slightly more symmetric.

The implementation of LBM to study stretching particle-laden liquid bridges provides a tool for understanding the detailed dynamics and how the particle interaction with the interface behaves. Our implementation is three-dimensional, with no assumption of axisymmetry. The finite size of the particles is explicitly resolved, allowing us to capture wetting properties and capillary forces on the particles. One drawback is that the diffuse interface formulation requires a separation of scales between the size of the interface and other relevant length scales of the problem; the interface thickness should be small relative to the particles, which in turn should be small relative to the initial bridge diameter. This necessitates a large domain, and results in an expensive computation. Since this code uses a fixed Cartesian grid, an adaptive mesh refinement approach would increase the computational efficiency. The behaviors reported in this paper are dependent on many factors including particle size relative to the bridge, fluid viscosity, and plate velocity. Further study is planned to gain insight into this and related problems in interfacial flows of mixtures.

\section{Acknowledgements}

Funding for this research was provided by the CCNY-University of Chicago MRSEC Partnership on the Dynamics of Heterogeneous and Particulate Materials at the City College of New York, an NSF PREM (Award Number DMR-0934206). Work at the University of Chicago was supported by the NSF MRSEC under DMR-0820054. This research was also supported, in part, by a grant of computer time from the City University of New York High Performance Computing Center under NSF Grants CNS-0855217, CNS-0958379, and ACI-1126113.

\section{References}

[1] É. Guazzelli \& J. Morris, A Physical Introduction to Suspension Dynamics. Cambridge: Cambridge University Press, 2012

[2] P.A. Kralchevsky \& K. Nagayama, "Capillary interactions between particles bound to interfaces, liquid films, and biomembranes" Advances in Colloid and Interface Science 85, 145-192 (2000)

[3] X. Zhao, J.R.G. Evans, M.J. Edirisinghe, and J.H. Song, "Ink-jet printing of ceramic pillar arrays" J. Materials Sci. 37, 1987-1992 (2002) 
[4] S. Daplyn \& L. Lin, "Evaluation of pigmented ink formulations for jet printing onto textile fabrics" Pigment and Resin Tech. vol. 32, no. 5, 307-318 (2003)

[5] S.H. Ko, H. Pan, C.P. Grigoropoulos, C.K. Luscombe, J.M.J. Frèchet, and D. Poulikakos, "All ink-jet-printed flexible electronics fabrication on a polymer substrate by low-temperature high-resolution selective laser sintering of metal nanoparticles" Nanotechnology 18, 345202 (2007)

[6] X. Wang, W.W. Carr, D.G. Bucknall, and J.F. Morris, "Drop-on-demand drop formation of colloidal suspensions" Int. J. Multiphase Flow 38, 17-26 (2012)

[7] A. Yusof, H. Keegan, C.D. Spillane, O.M. Sheils, C.M. Martin, J.J. O'Leary, R. Zengerle, and P. Koltay "Inkjet-like printing of single-cells" Lab Chip 11, 2447-2454 (2011)

[8] J. Eggers, "Nonlinear dynamics and breakup of free-surface flows" Rev. Mod. Phys. Vol. 69, No. 3, 865-929 (1997)

[9] D.T. Papageorgiou, "On the breakup of viscous liquid threads" Phys. Fluids 7, 1529 (1995)

[10] R.J. Furbank \& J.F. Morris, "An experimental study of particle effects on drop formation" Phys. Fluids vol. 16, no. 5, 1777-1790 (2004)

[11] R.J. Furbank \& J.F. Morris, "Pendant drop thread dynamics of particle-laden liquids" Int. J. Multiphase Flow 33, 448-468 (2006)

[12] C. Bonnoit, T. Bertrand, E. Clément, and A. Lindner, "Accelerated drop detachment in granular suspensions" Phys. Fluids 24, 043304 (2012)

[13] M.S. van Deen, T. Bertrand, N. Vu, D. Quéré, E. Clément, and A. Lindner, "Particles accelerate the detachment of viscous liquids" Rheol. Acta 52, 403-412 (2013)

[14] M.Z. Miskin \& H.M. Jaeger, "Droplet formation and scaling in dense suspensions" Proc. Nat. Academy Sci. vol. 109 no. 12, 4389-4394

[15] T. Bertrand, C. Bonnoit, E. Clément, and A. Lindner, "Dynamics of drop formation in granular suspensions: the role of volume fraction" Granular Matter 14, 169-174 (2012)

[16] M. Roché, H. Kellay, and H.A. Stone, "Heterogeneity and the role of normal stresses during the extensional thinning of non-Brownian shear-thickening fluids" Phys. Rev. Lett. 107, 134503 (2011) 
[17] M. Hameed \& J.F. Morris, "Breakup of a liquid jet containing solid particles: a singularity approach" J. Appl. Math vol. 70, no. 3, 885-900 (2009)

[18] C. McIlroy \& O.G. Harlen, "Modeling capillary break-up of particulate suspensions" Phys. Fluids 26, 033101 (2014)

[19] K. Connington, T. Lee, and J.F. Morris, "Interaction of fluid interfaces with immersed solid particles using the lattice Boltzmann method for liquid-gas-particle systems" Under Review

[20] A.J.C. Ladd, "Numerical simulations of particulate suspensions via a discretized Boltzmann equation. Part 2. Numerical results" J. Fluid Mech. 271, 311-339 (1994)

[21] T. Lee and L. Liu, "Lattice Boltzmann Simulations of Micron-Scale Drop Impact on Dry Surfaces" J. Comput. Phys. 229, 8045 (2010)

[22] J.W. Cahn, "Critical-Point Wetting” J. Chem. Phys. 66, 3667 (1977)

[23] T. Lee and L. Liu, "Wall Boundary Conditions in the Lattice Boltzmann Equation Method for Non-Ideal Gases" Phys. Rev. E 78, 017702 (2008)

[24] L. Liu \& T. Lee, "Wall free energy based polynomial boundary conditions for nonideal gas lattice Boltzmann equation" Int. J. Modern Phys. C Vol. 20, No. 11, 1749-1768 (2009)

[25] X. He and L.S. Luo, "A priori derivation of the lattice Boltzmann equation" Phys. Rev. E 55, R6333 (1997)

[26] T. Lee, "Effects of incompressibility on the elimination of parasitic currents in the lattice Boltzmann equation method for binary fluids" Comput. Math Appl. 58, 987 (2009)

[27] X. He, S.Chen, and G. Doolen, "A novel thermal model for the lattice Boltzmann method in the incompressible limit" J. Comput. Phys. 146, 282 (1998)

[28] L.S. Luo, "Unified theory of the lattice Boltzmann model for nonideal gases" Phys. Rev. Lett. 81, 1618 (1998)

[29] Y. Chen and S. Chen, "Dissipative and dispersive behavior of lattice-based models for hydrodynamics” Phys. Rev. E 61, 2712 (2000)

[30] X. He, S. Chen, and R. Zhang, "A lattice Boltzmann scheme for incompressible multiphase flow and its application in simulation of Rayleigh-Taylor instability" J. Comput. Phys. 152, 642 (1999) 
[31] T. Lee and C. Lin, "Pressure evolution lattice Boltzmann equation method for twophase flow with phase change" Phys. Rev. E 67, 056703 (2003)

[32] A.S. Joshi and Y. Sun, "Wetting dynamics and particle deposition for an evaporating colloidal drop: A lattice Boltzmann study" Phys. Rev. E 82, 041401 (2010)

[33] K. Connington, T. Lee, and J. Morris, "Interaction of fluid interfaces with immersed solid particles using the lattice Boltzmann method for large fluid-fluid density ratios" Under Review

[34] K. Connington \& T. Lee, "A review of spurious currents in the lattice Boltzmann method for multiphase flows" J. Mech. Sci. Tech. 26, Is. 12, 3857-3863 (2012)

[35] K.Connington \& T. Lee, "Lattice Boltzmann simulations of forced wetting transitions of drops on superhydrophobic surfaces" J. Comput. Phys. 250, 601-615 (2013)

[36] X. Zhang, R.S. Padgett, and O.A. Basaran, "Nonlinear deformation and breakup of stretching liquid bridges" J. Fluid Mech. 329, 207-245 (1996)

[37] J. Eggers, "Universal pinching of 3D axisymmetric free-surface flow" Phys. Rev. Lett. vol. 71 , no. $21,3458-3460$ (1993)

[38] J. Eggers \& E. Villermaux, "Physics of liquid jets" Rep. Prog. Phys. 71, 036601 (2008)

[39] I.M. Krieger, "Rheology of monodisperse lattices" Adv. Colloid Interface Sci. 2, 111 (1972)

[40] H. Stechemesser \& A.V. Nguyen, "Time of gas-solid-liquid three-phase contact expansion in flotation"

[41] H. Haddadi \& J. F. Morris "Microstructure and rheology of finite inertia suspensions" J. Fluid Mech. 749, 431-459 (2014)

[42] A. Würger "Curvature-induced capillary interaction of spherical particles at a liquid interface" Phys Rev E 74, 041402 (2006) 